\title{
Mixed finite element methods with convection stabilization for the large eddy simulation of incompressible turbulent flows
}

\author{
Oriol Colomés ${ }^{\mathrm{a}}$, Santiago Badia ${ }^{\mathrm{a}, \mathrm{b}}$, Javier Principe ${ }^{\mathrm{a}, \mathrm{b}}$ \\ ${ }^{a}$ Centre Internacional de Mètodes Numèrics a l'Enginyeria (CIMNE), Parc Mediterrani de la Tecnologia, UPC, Esteve \\ Terradas 5, 08860 Castelldefels, Spain (\{ocolomes,sbadia,principe\}@cimne.upc.edu). \\ ${ }^{b}$ Universitat Politècnica de Catalunya, Jordi Girona 1-3, Edifici C1, 08034 Barcelona, Spain.
}

\begin{abstract}
The variational multiscale method thought as an implicit large eddy simulation model for turbulent flows has been shown to be an alternative to the widely used physical-based models. This method is traditionally combined with equal-order velocity-pressure pairs, since it provides pressure stabilization. In this work, we consider a different approach, based on inf-sup stable elements and convection-only stabilization. In order to do so, we consider a symmetric projection stabilization of the convective term using a orthogonal subscale decomposition. The accuracy and efficiency of this method compared with residual-based algebraic subgrid scales and orthogonal subscales methods for equal-order interpolation is assessed in this paper. Moreover, when inf-sup stable elements are used, the grad-div stabilization term has been shown to be essential to guarantee accurate solutions. Hence, a study of the influence of such term in the large eddy simulation of turbulent incompressible flows is also performed. Furthermore, a recursive block preconditioning strategy has been considered for the resolution of the problem with an implicit treatment of the projection terms. Two different benchmark tests have been solved: the Taylor-Green Vortex flow with $R e=1600$, and the Turbulent Channel Flow at $R e_{\tau}=395$.
\end{abstract}

Keywords: Large eddy simulation, turbulence, variational multiscale, block recursive preconditioning, grad-div stabilization

\section{Introduction}

The simulation of incompressible turbulent flows is needed to solve many problems in science and engineering, e.g., flows around aircrafts, wind turbines, cars, bridges, etc. The computational fluid dynamics field is constantly evolving and searching the best way to achieve faster and cheaper flow simulations, taking advantage of the increasing computational power of supercomputers.

Turbulent flows are characterized by having multiple scales, both in space and time, that make their simulation a really challenging task. Direct Numerical Simulations (DNS) of turbulent flows are used to capture the physical phenomena at all scales, even the smallest ones, but they consume a large amount of computational resources. A technique that is increasingly used and that saves a lot of computational cost is the Large Eddy Simulation (LES), which basically consists in separating the flow in a coarse scale and a fine one, simulating the coarser and modelling the finer [1]. The model of the fine scales in a LES method can be developed by a physical approach, which is defined taking into account the physical phenomena that take place on the smallest scales, or by a purely numerical approach that does not introduce any modification to the governing equations at the continuous level. This last numerical approach is commonly denoted as Implicit LES (ILES), see for instance [2].

The Variational MultiScale (VMS) method introduced by Hughes in $[3,4]$ is a framework to develop stable and accurate numerical approximations of partial differential equations, preventing numerical instabilities that arise when the standard Galerkin Finite Element (FE) method is used. In the context of the incompressible Navier-Stokes equations there are two well known instabilities. First, there is the need to satisfy a compatibility between velocity and pressure approximation spaces, i.e., the inf-sup condition, which is not satisfied when equal order interpolation is used. Second, we have the instabilities that appear when convective dominated flows are simulated, which can be solved by adding some kind of extra dissipation to the problem, as in, e.g., [5]. There is also a much less known loss of accuracy in 
the discrete velocity due to poor mass conservation [6] which can be linked to a poor resolution of the pressure typical of mixed interpolations that satisfy the inf-sup condition [7].

The VMS method was firstly thought as an ILES method in $[8,9,10]$ and, since then, several VMS methods have been developed and used as ILES. We can distinguish between those that introduce a three scale decomposition into resolved large and small scales and unresolved scales [11, 12, 13, 14], with a Smagorinsky type model for the influence of unresolved scales onto the small resolved ones, and those that introduce a two scale decomposition into resolved and unresolved ones $[15,16]$ using a residual based or projection based model of the unresolved scales to account for their influence into the resolved ones.

In [17], a two scale VMS approach through Orthogonal SubScales (OSS) was firstly introduced. The main idea of the OSS method is to select the space of small scales orthogonal to the FE space, in contrast to the traditional choice of taking the subscales proportional to the residual, which is called Algebraic SubGrid Scale (ASGS) in [17]. Apart from the choice of the space of subscales, their time dependency and the VMS splitting of nonlinear terms was studied in [18]. Several combinations of these modelling possibilities where exhaustively assessed for homogeneous and wall bounded turbulent flows in [16]. In that work, an explicit algorithm to compute the orthogonal projections was used and the projection of the whole residual was considered.

An alternative definition of the OSS method was proposed in [19] using a term-by-term stabilization that does not involve the full residual. A similar term-by-term stabilization approach was followed in [20], where the Local Projection Stabilization (LPS) method was introduced. This type of techniques are also known as symmetric projection stabilization, and the key ingredient that leads to different schemes

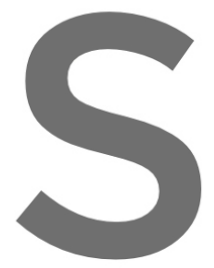

is the definition of such

is that one can avoid the (ISS) velocity-pressure projection can be easil equal-order or ISS pair the grad-div stabilization
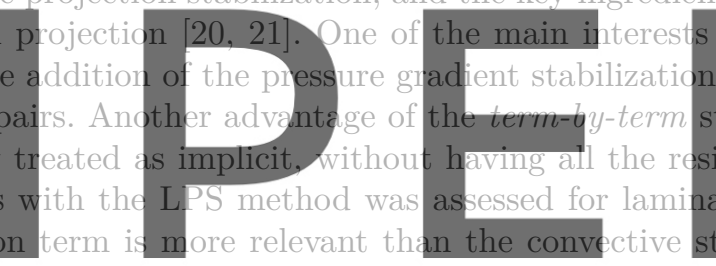

are used. The same conclusion was pointed out in

here the turbulent channel flow is studied

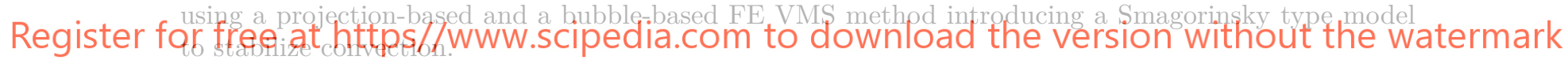

The main goal of this paper is to assess for the first time the accuracy and efficiency of convectionstabilized ISS schemes as ILES methods, where symmetric projection stabilization is used. In particular, we analyze the term-by-term OSS method with implicit treatment of the projection for turbulent flows. We also analyse the influence of the grad-div stabilization on the accuracy of the method. For ISS discretizations, the influence of this term on the mass conservation is well known [6] but it also influences the computational cost of the linear solvers [23, 24]. In this respect, we present a block preconditioning strategy that makes use of recursive block factorizations [25] to deal with the implicit projections and with the saddle point structure of the velocity-pressure coupling, which can also be applied to equal order interpolation with pressure stabilization. The comparison of the results is made with respect those obtained using the residual-based ASGS for which we also use a block preconditioning strategy.

This work is organized as follows. The Navier-Stokes equations together with some notation used in the paper are stated in Sect. 2. The VMS framework is introduced in Sect. 3, which includes the final discrete formulation of the ASGS method, given in Sect. 3.1, the term-by-term OSS given in Sect. 3.2, the term-by-term OSS with ISS elements given in Sect. 3.3 and also a brief discussion of known properties of the grad-div stabilization given in Sect. 3.4. The recursive block iterative strategy proposed to solve the linear system of the monolithic problem is presented in Sect. 4. The numerical results are shown in Sect. 5, where two different turbulent tests are analysed: the Taylor-Green Vortex flow in Sect. 5.2 and the Turbulent Channel Flow in Sect. 5.3. Finally, some conclusions are pointed out in Sect. 6 .

\section{Problem statement}

Let $\Omega$ be a bounded domain of $\mathbb{R}^{d}$, where $d=2,3$ is the number of space dimensions, $\Gamma=\partial \Omega$ its boundary and $(0, T]$ the time interval. The strong form of the steady Navier-Stokes problem consists in 
finding the velocity field $\mathbf{u}$ and the pressure field $p$ such that

$$
\begin{aligned}
\partial_{t} \mathbf{u}-\nu \Delta \mathbf{u}+\mathbf{u} \cdot \nabla \mathbf{u}+\nabla p & =\mathbf{f} & & \text { in } \Omega \times(0, T], \\
\nabla \cdot \mathbf{u} & =0 & & \text { in } \Omega \times(0, T],
\end{aligned}
$$

with $\mathbf{f}$ the force vector and $\nu$ the kinematic viscosity. Hereafter bold characters will denote vectors and tensors.

Equations (1) and (2) need to be supplied with appropriate boundary and initial conditions. The boundary $\Gamma$ is divided into the Dirichlet $\left(\Gamma_{D}\right)$ and the Neumann $\left(\Gamma_{N}\right)$ parts such that $\Gamma_{D} \cup \Gamma_{N}=\Gamma$ and $\Gamma_{D} \cap \Gamma_{N}=\%$. Then, the boundary and initial conditions can be written as

$$
\begin{array}{rlrl}
\mathbf{u} & =\mathbf{u}_{g} & & \text { on } \Gamma_{D} \times(0, T], \\
\left(-p \cdot \mathbf{I}+\nu\left(\nabla \mathbf{u}+\nabla \mathbf{u}^{T}\right)\right) \cdot \mathbf{n} & =\mathbf{t}_{N} & \text { on } \Gamma_{N} \times(0, T], \\
\mathbf{u}(x, 0) & =\mathbf{u}_{0}(x) & & \text { in } \Omega \times\{0\},
\end{array}
$$

n being the unit outward vector normal to $\Gamma$.

Some notation need to be stated in order to derive the weak form of the problem (1)-(5). We denote by $L^{p}(\Omega), 1 \leq p<\infty$, the spaces of functions such that their $p$-th power is absolutely integrable in $\Omega$. In particular, for the case in which $p=2$, we have a Hilbert space with scalar product

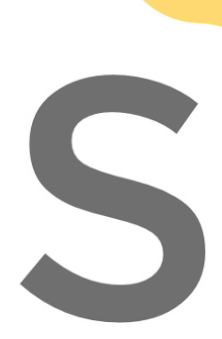

$(u, v)_{\Omega} \equiv(u, v):=\int u(x) v(x) d \Omega$

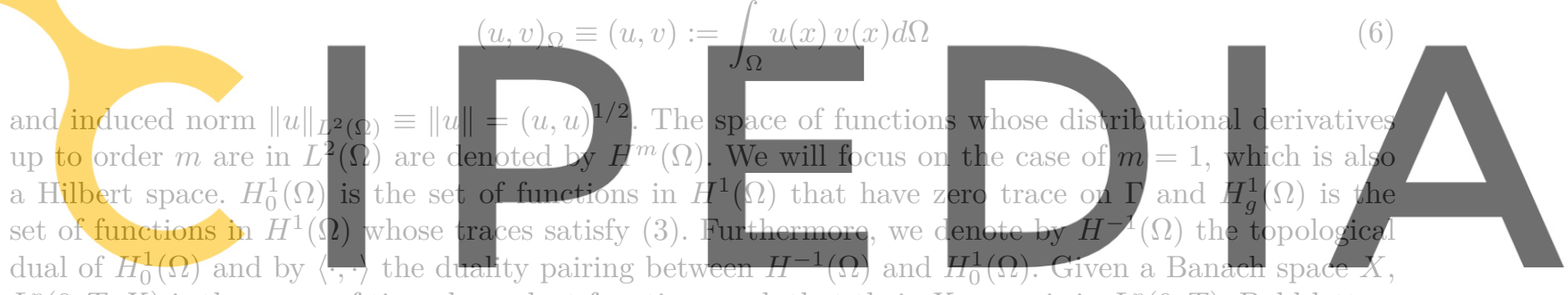

$L^{p}(0, T ; X)$ is the space of time-dependent functions such that their $X$-norm is in $L^{p}(0, T)$. Bold letters

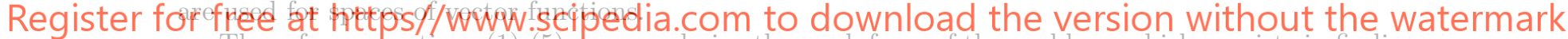

Then, from equations (1)-(5) one can derive the weak form of the problem, which consists in finding $[\mathrm{u}, p] \in \mathbb{L}^{2}\left(0, T ; \mathcal{V}_{g}\right) \times L^{1}\left(0, T ; \mathcal{Q}_{0}\right)$ such that

$$
\left(\partial_{t} \mathbf{u}, \mathbf{v}\right)+B(\mathbf{u},(\mathbf{u}, p),(\mathbf{v}, q))=\langle\mathbf{f}, \mathbf{v}\rangle \quad \forall \mathbf{v} \in \mathcal{V}_{0}, \quad \forall q \in \mathcal{Q}_{0},
$$

satisfying the initial condition (5) in a weak sense. Here $\mathcal{V}_{0}:=\mathbf{H}_{0}^{1}(\Omega), \mathcal{V}_{g}:=\mathbf{H}_{g}^{1}(\Omega)$ and $\mathcal{Q}_{0}:=L^{2}(\Omega) / \mathbb{R}$ and the form $B(\mathbf{u},(\mathbf{u}, p),(\mathbf{v}, q))$ is defined as

$$
B(\mathbf{u},(\mathbf{u}, p),(\mathbf{v}, q)):=\nu(\nabla \mathbf{u}, \nabla \mathbf{v})+b(\mathbf{u}, \mathbf{u}, \mathbf{v})-(p, \nabla \cdot \mathbf{v})+(q, \nabla \cdot \mathbf{u})
$$

with the trilinear form of the convective term $b(\mathbf{u}, \mathbf{v}, \mathbf{w})$ defined in its skew symmetric version

$$
b(\mathbf{u}, \mathbf{v}, \mathbf{w})=\frac{1}{2}(\mathbf{u} \cdot \nabla \mathbf{v}, \mathbf{w})-\frac{1}{2}(\mathbf{v}, \mathbf{u} \cdot \nabla \mathbf{w})+\frac{1}{2}(\mathbf{v},(\mathbf{u} \cdot \mathbf{n}) \mathbf{w})_{\Gamma_{N}} .
$$

\section{VMS methods}

Let us consider a FE partition $\mathcal{T}_{h}$ of the domain $\Omega$ from which we can construct conforming finite dimensional spaces for the velocity $\mathcal{V}_{0, h} \subset \mathcal{V}_{0}, \mathcal{V}_{g, h} \subset \mathcal{V}_{g}$, and for the pressure $\mathcal{Q}_{0, h} \subset \mathcal{Q}_{0}$. The Galerkin FE approximation of the problem (7) consists in finding $\left[\mathbf{u}_{h}, p_{h}\right] \in \mathbf{L}^{2}\left(0, T ; \mathcal{V}_{g, h}\right) \times L^{1}\left(0, T ; \mathcal{Q}_{0, h}\right)$ such that

$$
\left(\partial_{t} \mathbf{u}_{h}, \mathbf{v}_{h}\right)+B\left(\mathbf{u}_{h},\left(\mathbf{u}_{h}, p_{h}\right),\left(\mathbf{v}_{h}, q_{h}\right)\right)=\left\langle\mathbf{f}, \mathbf{v}_{h}\right\rangle \quad \forall \mathbf{v}_{h} \in \mathcal{V}_{0, h}, \forall q_{h} \in \mathcal{Q}_{0, h} .
$$

In order to overcome the numerical instabilities and, eventually, to bypass the inf-sup condition that arises when problem (10) is solved, we use the VMS approach $[3,4]$ which consists in a two-scale decomposition of spaces $\mathcal{V}_{0}, \mathcal{V}_{g}$ and $\mathcal{Q}_{0}$ as $\mathcal{V}_{0}=\mathcal{V}_{0, h} \oplus \widetilde{\mathcal{V}}_{0}, \mathcal{V}_{g}=\mathcal{V}_{g, h} \oplus \widetilde{\mathcal{V}}_{g}$ and $\mathcal{Q}=\mathcal{Q}_{0, h} \oplus \widetilde{\mathcal{Q}}_{0}$, where $\widetilde{\mathcal{V}}_{0}$, $\widetilde{\mathcal{V}}_{g}$ and $\widetilde{\mathcal{Q}}_{0}$ are infinite-dimensional spaces that complete the FE spaces in $\mathcal{V}_{0}, \mathcal{V}_{g}$ and $\mathcal{Q}_{0}$, respectively. 
Hereinafter the subscript $(\cdot)_{h}$ will denote the FE component and the tilde $\widetilde{(\cdot)}$ the subgrid component. Applying the two-scale decomposition to (7) we obtain

$$
\left(\partial_{t} \mathbf{u}_{h}, \mathbf{v}_{h}\right)+\left(\partial_{t} \tilde{\mathbf{u}}, \mathbf{v}_{h}\right)+B\left(\mathbf{u} ;\left[\mathbf{u}_{h}, p_{h}\right],\left[\mathbf{v}_{h}, q_{h}\right]\right)-\left(\tilde{\mathbf{u}}, \nu \Delta \mathbf{v}_{h}+\mathbf{a} \cdot \nabla \mathbf{v}_{h}+\nabla q_{h}\right)_{h}-\left(\tilde{p}, \nabla \cdot \mathbf{v}_{h}\right)=\left\langle\mathbf{f}, \mathbf{v}_{h}\right\rangle,
$$

where $(\cdot, \cdot)_{h}=\sum_{K \in \mathcal{T}_{h}}(\cdot, \cdot)_{K}$ is the sum of scalar products (6) over each element $K$ of the partition $\mathcal{T}_{h}$. The terms involving the subscales come from an element-wise integration by parts, in which the boundary terms $\left(\mathbf{v}_{h}, \nu \mathbf{n} \cdot \nabla \tilde{\mathbf{u}}\right)_{\partial h}$ and $\left(q_{h}, \mathbf{n} \cdot \tilde{\mathbf{u}}\right)_{\partial h}$ have been neglected (the subscript $\partial h$ is used to denote the sum over all elements of the integral on the boundary of each element). It also involves the approximation $b\left(\mathbf{a}, \tilde{\mathbf{u}}, \mathbf{u}_{h}\right) \approx-\left(\tilde{\mathbf{u}}, \mathbf{a} \cdot \nabla \mathbf{v}_{h}\right)$ which implies neglecting $\left(\mathbf{v}_{h}, \mathbf{n} \cdot \mathbf{a} \tilde{\mathbf{u}}\right)_{\partial h}$ and $\left(\tilde{\mathbf{u}}, \nabla \cdot \mathbf{a} \mathbf{v}_{h}\right)$. These approximations are discussed in [18] together with the choice of a which defines the type of scale splitting (linear or nonlinear), see also [16].

Problem (11) depends on $\tilde{\mathbf{u}} \in \widetilde{\mathcal{V}}_{0}$ and on $\tilde{p} \in \widetilde{\mathcal{Q}}_{0}, \widetilde{\mathcal{V}}_{0}$ and $\widetilde{\mathcal{Q}}_{0}$ being infinite-dimensional. Therefore, the equations for $\tilde{u}$ and $\tilde{p}$ obtained after applying the two-scale decomposition cannot be directly solved, but some modelling steps are needed to obtain a feasible method. In this work we consider the velocity subscale as linear $\left(\mathbf{a}=\mathbf{u}_{h}\right)$ and quasi-static, while the pressure subscale is neglected when equal order interpolation is used. Different approaches could be used for the definition of the subscales, see [16] for a deep explanation of the different choices and their numerical evaluation with equal order approximation. Approximating the Navier-Stokes operators by the stabilization parameters $\tau_{m}^{-1}$ and $\tau_{c}^{-1}$ (see for example $[18]$ ), the fine scale problem can be written as
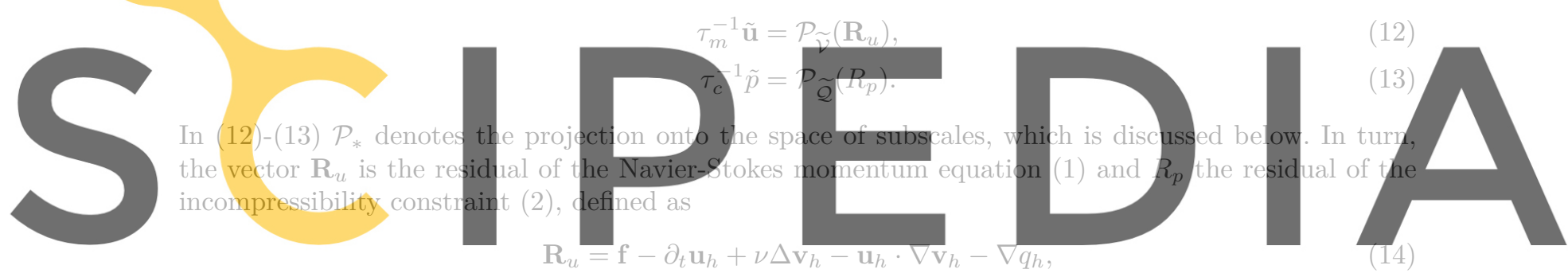

Register for

$R_{p_{0}}=-\nabla \cdot \mathbf{u}_{h}$

$(15)$

Finally, the expressions of the stabilization parameters $\tau_{m}$ and $\tau_{c}$ are

$$
\begin{aligned}
& \tau_{m}=\left(\frac{c_{1} \nu}{h^{2}}+\frac{c_{2}\left|\mathbf{u}_{h \mid}\right|}{h}\right)^{-1} \\
& \tau_{c}=\frac{h^{2}}{c_{1} \tau_{m}}
\end{aligned}
$$

where $h$ is the mesh size and $c_{1}$ and $c_{2}$ are algorithmic constants. The choice of the value for $c_{1}$ and $c_{2}$ make a significant difference on the VMS method, specially for turbulent flows, where the energy dissipation introduced by the stabilization method takes an important role in the final solution. In this work, we select $c_{1}=12$ and $c_{2}=2$, following [16]. Introducing $\tilde{\mathbf{u}}$ and $\tilde{p}$ into (11) we get the final discrete problem

$$
\left(\partial_{t} \mathbf{u}_{h}, \mathbf{v}_{h}\right)+B_{h}\left(\mathbf{u}_{h},\left(\mathbf{u}_{h}, p_{h}\right),\left(\mathbf{v}_{h}, q_{h}\right)\right)=L_{h}\left(\mathbf{v}_{h}, q_{h}\right) \quad \forall \mathbf{v}_{h} \in \mathcal{V}_{0, h}, \forall q_{h} \in \mathcal{Q}_{0, h},
$$

The bilinear form $B_{h}$ and the linear form $L_{h}$ depend on the particular VMS method as discussed below.

\subsection{Residual-based $A S G S$}

The space for the subscales $\widetilde{\mathcal{V}}_{0}$ is determined by the definition of the projection $\mathcal{P}$ appearing in the right-hand side of (12)-(13). The ASGS method is obtained taking the subscales in the space of the residuals, that is, $\mathcal{P}_{\widetilde{\mathcal{V}}}:=\mathbf{I}$ and $\mathcal{P}_{\widetilde{\mathcal{Q}}}:=I$. The final discrete is given by (18) with $B_{h}=B_{\text {asgs }}$ and $L_{h}=L_{\text {asgs }}$ given by

$$
\begin{aligned}
B_{\text {asgs }}\left(\mathbf{u}_{h},\left(\mathbf{u}_{h}, p_{h}\right),\left(\mathbf{v}_{h}, q_{h}\right)\right) & :=B\left(\mathbf{u}_{h},\left(\mathbf{u}_{h}, p_{h}\right),\left(\mathbf{v}_{h}, q_{h}\right)\right) \\
& +\left(\tau_{m}\left(\partial_{t} \mathbf{u}_{h}-\nu \Delta \mathbf{u}_{h}+\mathbf{u}_{h} \cdot \nabla \mathbf{u}_{h}+\nabla p_{h}\right), \nu \Delta \mathbf{v}_{h}+\mathbf{u}_{h} \cdot \nabla \mathbf{v}_{h}+\nabla q_{h}\right), \\
L_{\text {asgs }}\left(\mathbf{v}_{h}, q_{h}\right) & :=\left\langle\mathbf{f}, \mathbf{v}_{h}\right\rangle+\left(\tau_{m} \mathbf{f}, \nu \Delta \mathbf{v}_{h}+\mathbf{u}_{h} \cdot \nabla \mathbf{v}_{h}+\nabla q_{h}\right) .
\end{aligned}
$$


Note that the pressure subscale term has been neglected in (19), that is we have taken $\tau_{c}=0$. We have observed in [16] (using equal order interpolation) that this term introduces extra dissipation and does not in general improve the solution significantly.

\subsection{Term by term OSS}

Another possibility introduced in [17] is to consider the space of the subscales orthogonal to the FE space. The main motivation of the method is that a stability estimate for the projection onto the FE space of the pressure and the convective terms can already be obtained in the standard Galerkin method and therefore the only "missing" part is the orthogonal one. The Orthogonal Subscales (OSS) method is then obtained taking $\mathcal{P}_{\widetilde{\mathcal{V}}}:=\Pi_{h}^{\perp}=\mathbf{I}-\Pi_{h}$ where $\Pi_{h}$ is a projection onto the FE space. The $L^{2}$ orthogonality between the $\mathrm{FE}$ and subscale spaces is guaranteed considering the $\tau_{m}$-weighted projection

$$
\left(\tau_{m} \Pi_{h}(\mathrm{w}), \mathrm{v}_{h}\right)=\left(\tau_{m} \mathbf{w}, \mathbf{v}_{h}\right) \quad \forall \mathbf{v}_{h} \in \mathcal{V}_{0, h}
$$

which requires the solution of a linear system defined by a $\tau_{m}$-weighted mass matrix. Note that for this choice, the residual of the momentum equation does not depend on $\partial_{t} \mathbf{u}_{h}$. Likewise, $\mathcal{P}(\mathbf{f})$ in this case is only well defined for $\mathbf{f} \in L^{2}(\Omega)^{d}$. In the case of minimum regularity, $\mathbf{f} \in H^{-1}(\Omega)^{d}$, this term can be simply neglected without upsetting the accuracy of the method.

Remark 3.1. Alternatively we can consider the standard $L^{2}$ projection
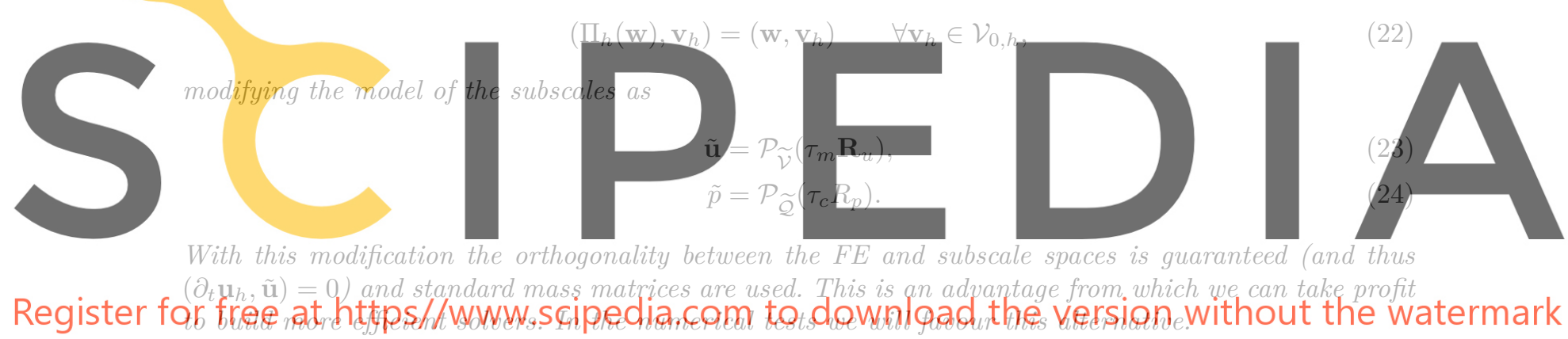

Neglecting the pressure subscales the OSS method is given by (18) with $B=B_{\text {oss }}$ and $L_{h}\left(\mathbf{v}_{h}, q_{h}\right)=$ $\left\langle\mathbf{f}, \mathbf{v}_{h}\right\rangle$ where

$$
\begin{aligned}
B_{\text {oss }}\left(\mathbf{u}_{h},\left(\mathbf{u}_{h}, p_{h}\right),\left(\mathbf{v}_{h}, q_{h}\right)\right) & =B\left(\mathbf{u}_{h},\left(\mathbf{u}_{h}, p_{h}\right),\left(\mathbf{v}_{h}, q_{h}\right)\right) \\
& +\left(\tau_{m}\left(-\nu \Delta \mathbf{u}_{h}+\mathbf{u}_{h} \cdot \nabla \mathbf{u}_{h}+\nabla p_{h}\right), \nu \Delta \mathbf{v}_{h}+\mathbf{u}_{h} \cdot \nabla \mathbf{v}_{h}+\nabla q_{h}\right) \\
& -\left(\tau_{m} \boldsymbol{\eta}_{h}, \nu \Delta \mathbf{v}_{h}+\mathbf{u}_{h} \cdot \nabla \mathbf{v}_{h}+\nabla q_{h}\right) .
\end{aligned}
$$

where $\boldsymbol{\eta}_{h}:=\Pi_{h}\left(\mathbf{R}_{u}\right)$ is computed solving

$$
\left(\tau_{m} \boldsymbol{\eta}_{h}, \boldsymbol{\kappa}_{h}\right)+\left(\tau_{m}\left(-\nu \Delta \mathbf{u}_{h}+\mathbf{u}_{h} \cdot \nabla \mathbf{u}_{h}+\nabla p_{h}\right), \boldsymbol{\kappa}_{h}\right)=\left(\tau_{m} \mathbf{f}, \boldsymbol{\kappa}_{h}\right) \quad \forall \boldsymbol{\kappa}_{h} \in \mathcal{V}_{h, 0},
$$

An implicit implementation of this method would require the introduction of an extra variable (the projection $\boldsymbol{\eta}_{h}$ ) and, more importantly, this variable would be coupled with both velocity and pressure. Due to the Laplacian terms in the formulation, the velocity-projection coupling is non-symmetric, as it can be seen in (25) and (26).

In order to reduce the coupling between variables we consider the term by term OSS method proposed in [19]. The main goal of this alternative is to stabilize separately the convective term and the pressure gradient term by two uncoupled orthogonal projections. As noted in [19] the term by term OSS has better stability properties than the classical one. Considering quasi-static and linear subscales the discrete problem is given by (18) with $B=B_{t b t \_o s s}$ and $L_{h}\left(\mathbf{v}_{h}, q_{h}\right)=\left\langle\mathbf{f}, \mathbf{v}_{h}\right\rangle$ where

$$
\begin{aligned}
B_{\text {tbt_oss }}\left(\mathbf{u}_{h},\left(\mathbf{u}_{h}, p_{h}\right),\left(\mathbf{v}_{h}, q_{h}\right)\right) & =B\left(\mathbf{u}_{h},\left(\mathbf{u}_{h}, p_{h}\right),\left(\mathbf{v}_{h}, q_{h}\right)\right) \\
& +\left(\tau_{m} \mathbf{u}_{h} \cdot \nabla \mathbf{u}_{h}, \mathbf{u}_{h} \cdot \nabla \mathbf{v}_{h}\right)+\left(\tau_{m} \nabla p_{h}, \nabla q_{h}\right) \\
& -\left(\tau_{m} \boldsymbol{\eta}_{h}, \mathbf{u}_{h} \cdot \nabla \mathbf{v}_{h}\right)-\left(\tau_{m} \boldsymbol{\xi}_{h}, \nabla q_{h}\right) .
\end{aligned}
$$


and $\boldsymbol{\eta}_{h}:=\Pi_{h}\left(\mathbf{u}_{h} \cdot \nabla \mathbf{u}_{h}\right)$ and $\boldsymbol{\xi}_{h}:=\Pi_{h}\left(\nabla p_{h}\right)$ are computed solving

$$
\begin{aligned}
& \left(\tau_{m} \boldsymbol{\eta}_{h}, \boldsymbol{\kappa}_{h}\right)=\left(\tau_{m} \mathbf{u}_{h} \cdot \nabla \mathbf{u}_{h}, \boldsymbol{\kappa}_{h}\right) \\
& \forall \kappa_{h} \in \mathcal{V}_{h, 0}, \\
& \left(\tau_{m} \boldsymbol{\xi}_{h}, \boldsymbol{\zeta}_{h}\right)=\left(\tau_{m} \nabla p_{h}, \boldsymbol{\zeta}_{h}\right) \\
& \forall \boldsymbol{\zeta}_{h} \in \mathcal{V}_{h, 0} .
\end{aligned}
$$

Note that with the term by term OSS method with implicit FE projections, there are $3 d+1$ unknowns per node, while for ASGS the number of unknowns is $d+1$ per node. A priori it seems that such increase of unknowns make the former method not appealing in front of ASGS, but we will see later that the increase of computational cost is not linear with the increase of unknowns in this case. Furthermore, an optimal block preconditioning technique can be used to solve problem (18)-(27)-(28)-(29) taking advantage of its block structure. This point is further discussed in Sect. 4.

\subsection{Term by term OSS with ISS elements}

For equal order interpolation the pressure stabilization is mandatory but for ISS FE spaces pressure stability is guaranteed. Then, we could define a term by term OSS method for ISS FE that only stabilizes the convective term by an orthogonal FE projection. This approach would reduce the number of unknowns per node, keeping stability properties and the better conditioned matrix than ASGS. The definition of the term by term OSS-ISS method can be given by equation (18) with $B=B_{\text {tbt_oss_iss }}$ and $L_{h}\left(\mathbf{v}_{h}, q_{h}\right)=\left\langle\mathbf{f}, \mathbf{v}_{h}\right\rangle$ where

$$
B_{\text {tbt_oss_iss }}\left(\mathbf{u}_{h},\left(\mathbf{u}_{h}, p_{h}\right),\left(\mathbf{v}_{h}, q_{h}\right)\right)=B\left(\mathbf{u}_{h},\left(\mathbf{u}_{h}, p_{h}\right),\left(\mathbf{v}_{h}, q_{h}\right)\right)
$$
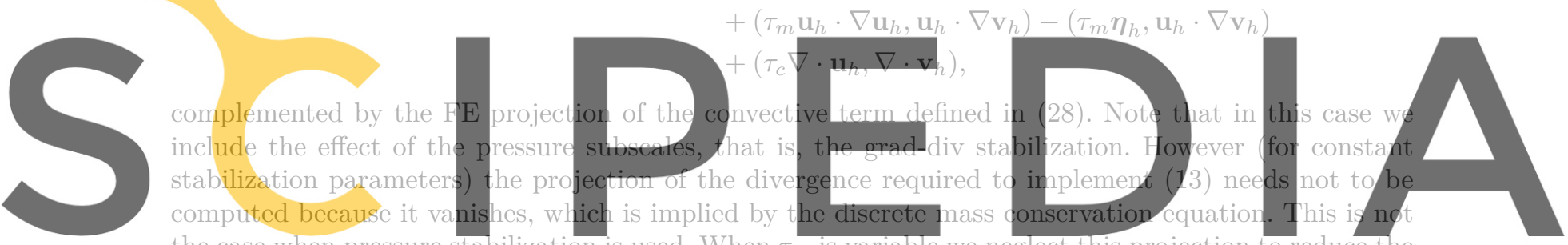

the case when pressure stabilization is used. When $\tau_{m}$ is variable we neglect this projection to reduce the computational cost. Note, however, that this approximation does not introduce any consistency error.

\subsection{The grad-div stabilization}

It is important to highlight here the presence of the pressure subscale term, $\tau_{c}\left(\nabla \cdot \mathbf{u}_{h}, \nabla \cdot \mathbf{v}_{h}\right)$ in $(30)$,

first introduced in [26]. Even though it is usually included in VMS formulations it is sometimes neglected in practice, particularly when equal order interpolations with pressure stabilization are considered [16]. As it is shown in [22], using the grad-div stabilization in the simulation of laminar flows produces a small improvement of the results obtained with equal order interpolation but a clear improvement of the results obtained with ISS elements.

In the VMS decomposition, this term comes from the residual of the incompressibility constraint and its addition improves the conservation of mass as well as the effect that the error on the pressure field produces on the velocity field. In [7, 27] the authors assessed the use of ISS elements for the incompressible Navier-Stokes equations in the laminar regime, highlighting the importance of this term. The optimal choice of the parameter $\tau_{c}$, discussed in [28], depends on the relative norms of the velocity and pressure (is therefore problem dependent) and can be of order one but also much bigger. On the other hand, in [29] it is proved that on a regular mesh, the Taylor-Hood approximations converge to a point-wise divergence-free solution, the one obtained using Scott-Vogelius (SV) elements [30], as $\tau_{c} \rightarrow \infty$. Then, it is seen that the optimal value of $\tau_{c}$ is an open question and, as stated in [7], we may consider the search of optimal parameters as a trade-off between mass and momentum balance in the FE system. In this work we will try to evaluate the importance of such term for turbulent incompressible flows when ISS elements are used. Thus, a detailed discussion of which are the values that should take $\tau_{c}$ is considered for each numerical test in further sections.

Apart from its influence on mass conservation, this term is also known to introduce numerical dissipation both when equal order [16] or ISS elements are used [7]. An energy balance of the term-byterm OSS is obtained taking $v_{h}=u_{h}$ and $q_{h}=p_{h}$ in (18) and using (30)

$$
\frac{1}{2} \frac{d}{d t}\left\|u_{h}\right\|^{2}+\nu\left\|\nabla \mathbf{u}_{h}\right\|^{2}+\left\|\tau_{m}^{1 / 2}\left(\mathbf{u}_{h} \cdot \nabla \mathbf{u}_{h}-\boldsymbol{\eta}_{h}\right)\right\|^{2}+\left\|\tau_{c}^{1 / 2} \nabla \cdot \mathbf{u}_{h}\right\|^{2}=\left\langle\mathbf{f}, \mathbf{u}_{h}\right\rangle .
$$


Apart from the viscous dissipation coming from the Galerkin method, which is negligible in turbulent flows, we get extra dissipation that comes from the control of the orthogonal projection of the convective term and the dissipation that comes from the grad-div stabilization. When equal order interpolations are used this extra dissipation is not necessary and very good results are obtained taking $\tau_{c}=0$ [16]. As discussed above, for ISS discretizations, the numerical dissipation introduced by the grad-div term is crucial to obtain accurate solutions. For instance, given a velocity space, the Taylor-Hood element has one order less pressure space than the stabilized equal order counterpart, leading to a poorer approximation of the mass conservation equation. Note that when $\tau_{c}$ goes to zero no dissipation is introduced but the accuracy in the satisfaction of mass conservation is poor. On the other hand taking $\tau_{c}$ large results in a strong imposition of the mass conservation (it acts as a penalty term) giving, for the Taylor-Hood pair on regular meshes, exact (point-wise) zero divergence in the limit [29]. In this case it turns out that the extra dissipation also vanishes as $\left\|\tau_{c}^{1 / 2} \nabla \cdot \mathbf{u}_{h}\right\|^{2} \longrightarrow 0$ in the limit of $\tau_{c} \longrightarrow \infty$ as $\tau_{c}^{-1 / 2}$ [31]. In any case it is important to keep in mind that when $\tau_{c}$ is changed the velocity field changes and the other dissipative terms in (31) also change. We cannot therefore conclude whether the method is more or less dissipative looking only at $\left\|\tau_{c}^{1 / 2} \nabla \cdot \mathbf{u}_{h}\right\|^{2}$. It is possible to arrive to such a conclusion when this parameter does not influences very much the solution, as in the case of equal order interpolation. This point is discussed in detail when presenting the numerical results in Sect. 5.

Finally, the grad-div term also has a strong influence on the conditioning of the linear system and therefore on the convergence of iterative solvers. When the monolithic system is considered, this term acts as an augmented Lagrangian term improving the convergence of block iterative schemes but it is

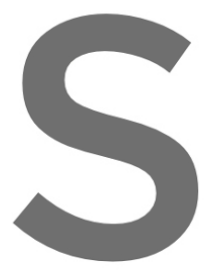
also known that it intraduces stifness in the veloci
An alternative to the parametcr truing need
divergence-free FEs that also satisfy the inf-sup co
stabilization vanishes). One of this group of elem
by the triangular/tetrahedral elements $P_{k} /$ pisc
except that the pressure space is discontinuous, w free (taking the pressule est function as the dive
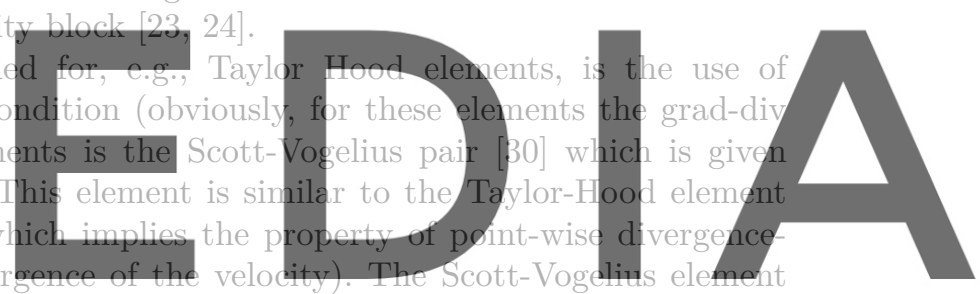

is ISS under mild assumptions on the mesh, e.g., the order of the interpolation $k \geq d$ and the mesh divergence-free have a different interpolation space for each velocity component, which for the 3D case can be stated as $Q_{k+1, k, k} \times Q_{k, k+1, k} \times Q_{k, k, k+1} / Q_{k}^{\text {disc }}$. In this case, the pressure field is also discontinuous with spurious modes filtered. We do not consider these approaches here.

\section{Block preconditioning for the monolithic problem}

A common approach when the OSS method is used is to treat the projection explicitly. That means to compute its value after the resolution of the velocity-pressure system and iterating until the solution converges. Although the resulting matrix has a better condition number than the ASGS method, the increase of nonlinear iterations due to the explicit treatment of the orthogonal projection may cause a lose of efficiency of this method in many cases. In [16] there is a computational cost analysis of these methods for turbulent incompressible flows where this effect can be shown.

Alternatively, the implicit approach of the OSS method increases the number of unknowns of the problem, not only having the usual velocity and pressure unknowns, but also the FE projection. Treating the FE projection as a new unknown, the system of equations to be solved is increased with equation (21) or (22). This projection unknown is coupled with both the velocity and the pressure and these blocks are non-symmetric which makes the application of the block preconditioning technique more difficult. As mentioned, this is not the case when the term by term OSS or the term by term OSS with ISS elements are considered.

In order to present our recursive block-preconditioning technique, let us assume that we solve the Navier-Stokes problem using (to fix ideas) a backward Euler time integration with a monolithic approach, i.e., without any velocity-pressure segregation algorithm. We consider the implicit term-by-term OSS stabilization given by the bilinear form (27) as this is the more general case when talking about number of unknowns that appear in the system, since there are the velocity, pressure and two projections. The 
case of the term by term OSS with ISS elements and the ASGS are obtained just eliminating rows and columns of this system.

Assuming that $\mathbf{u}_{h}, p_{h}, \boldsymbol{\eta}_{h}$ and $\boldsymbol{\xi}_{h}$ are defined by a FE interpolation from the nodal values $\left\{\mathbf{U}^{a}\right\}_{a=1, \ldots, N_{u}},\left\{P^{b}\right\}_{b=1, \ldots, N_{p}},\left\{\boldsymbol{\Upsilon}^{l}\right\}_{l=1, \ldots, N_{\eta}}$ and $\left\{\boldsymbol{\Xi}^{m}\right\}_{m=1, \ldots, N_{\xi}}$, the FE approximation of the velocity, pressure and projection fields can be written as

$$
\mathbf{u}_{h}(x)=\sum_{a=1}^{N_{u}} \boldsymbol{\phi}_{a}(x) \mathbf{U}^{a}, \quad p_{h}(x)=\sum_{b=1}^{N_{p}} \psi_{b}(x) P^{b}, \quad \boldsymbol{\eta}_{h}(x)=\sum_{l=1}^{N_{\eta}} \boldsymbol{\phi}_{\eta}(x) \boldsymbol{\Upsilon}^{l}, \quad \boldsymbol{\xi}_{h}(x)=\sum_{m=1}^{N_{\xi}} \boldsymbol{\phi}_{\xi}(x) \boldsymbol{\Xi}^{m},
$$

where $\left\{\boldsymbol{\phi}_{a, i}\right\}_{a=1, \ldots, N_{u} ; i=1, \ldots, d},\left\{\psi_{b}\right\}_{b=1, \ldots, N_{p}},\left\{\boldsymbol{\phi}_{l, i}\right\}_{l=1, \ldots, N_{\eta} ; i=1, \ldots, d}$ and $\left\{\boldsymbol{\phi}_{m, i}\right\}_{m=1, \ldots, N_{\xi} ; i=1, \ldots, d}$ are the Lagrangian basis associated to $\mathcal{V}_{h}$ and $\mathcal{Q}_{h} . N_{u}, N_{p}, N_{\eta}$ and $N_{\xi}$ are the total amount of nodes for the velocity, pressure and projection fields.

The matrix form of the problem (18) with the bilinear form (27) and, the projections (28)-(29) can be written as

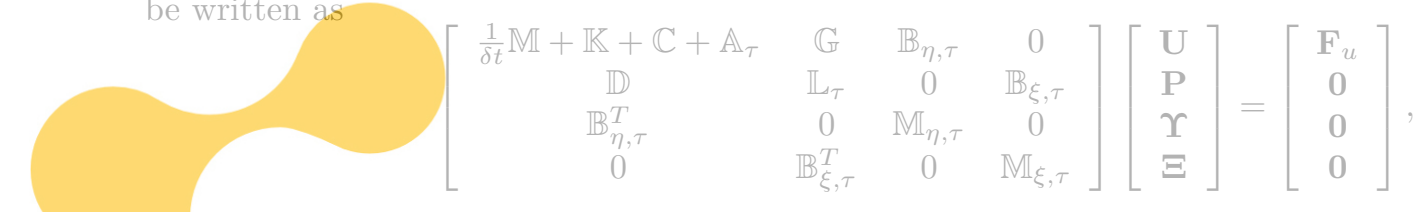

where $\mathbb{M}, \mathbb{K}, \mathbb{C}, \mathbb{D}$ and $\mathbb{G}$ are the matrices that arise from the Galerkin integration of the mass, diffusive, convective, velocity divergence and pressure gradient terms, respectively. The definition of the remaining

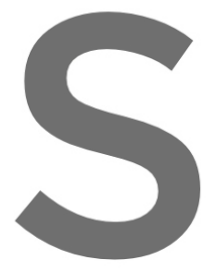
terms are given by
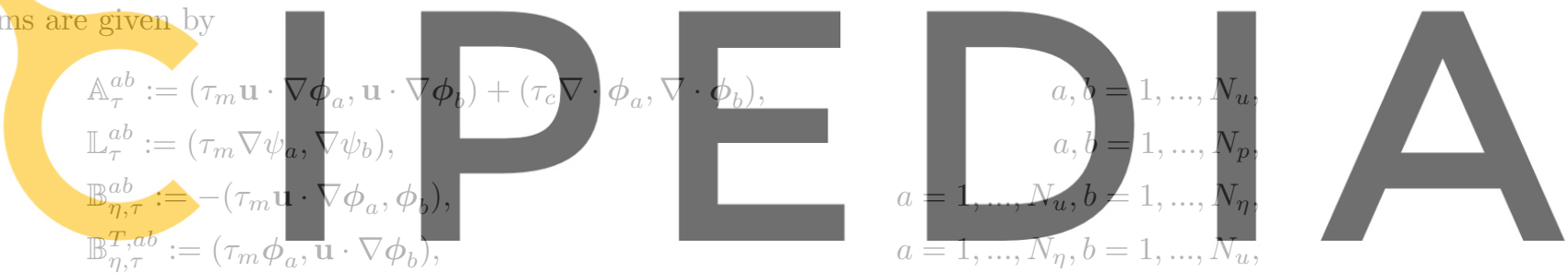

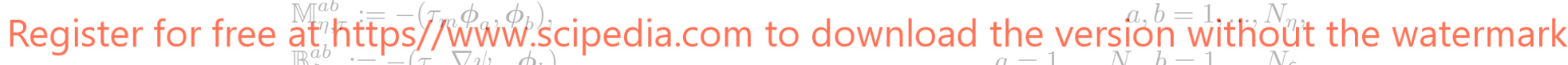 $\mathbb{B}_{\xi, \tau}^{a b}:=-\left(\tau_{m} \nabla \psi_{a}, \phi_{b}\right)$$$
\begin{aligned}
& \mathbb{B}_{\xi, \tau}^{T, a b}:=\left(\tau_{m} \phi_{a}, \nabla \psi_{b}\right) \\
& \mathbb{M}_{\xi, \tau}^{a b}:=-\left(\tau_{m} \boldsymbol{\phi}_{a}, \boldsymbol{\phi}_{b}\right),
\end{aligned}
$$$$
a=1, \ldots, N_{\xi}, b=1, \ldots, N_{p},
$$
$a, b=1, \ldots, N_{\xi}$,

being $a$ and $b$ the node identification. Note that $\mathbb{D}=-\mathbb{G}^{T}$, when Dirichlet boundary conditions are considered. In general, $N_{\xi}=N_{\eta}$, then, $\mathbb{M}_{\xi, \tau}=\mathbb{M}_{\eta, \tau}$.

For the ASGS method, only the first two rows and columns of the matricial system (32) are present, with different definitions of $\mathbb{A}_{\tau}$ and $\mathbb{L}_{\tau}$ that are straight forward from the bilinear form (19). In the case of OSS-ISS method, only the last row and column disappear, keeping the same definition for the remaining terms.

To solve the system (32) we use a recursive block-preconditioning technique. This methodology was used in [25] for a multiphysics problem like the thermally coupled inductionless MHD. The idea is to construct recursively block preconditioners of size $2 \times 2$ from an incomplete block factorization of the original $2 \times 2$ block matrices.

Let us consider a block system equivalent to (32) defined as

$$
\left[\begin{array}{cc}
\tilde{\mathbb{M}}_{\tau} & \tilde{\mathbb{B}}_{\tau}^{T} \\
\tilde{\mathbb{B}}_{\tau} & \tilde{\mathbb{K}}_{\tau}
\end{array}\right]\left[\begin{array}{c}
\tilde{\Xi} \\
\tilde{\mathbf{U}}
\end{array}\right]=\left[\begin{array}{c}
\mathbf{0} \\
\tilde{\mathbf{F}}_{u}
\end{array}\right]
$$

with $\tilde{\mathbb{K}}_{\tau}:=\left[\begin{array}{cc}\frac{1}{\delta t} \mathbb{M}+\mathbb{K}+\mathbb{C}+\mathbb{A}_{\tau} & \mathbb{G} \\ \mathbb{D} & \mathbb{L}_{\tau}\end{array}\right], \tilde{\mathbb{B}}_{\tau}:=\left[\begin{array}{cc}\mathbb{B}_{\eta, \tau} & 0 \\ 0 & \mathbb{B}_{\xi, \tau}\end{array}\right], \tilde{\mathbb{B}}_{\tau}^{T}:=\left[\begin{array}{cc}\mathbb{B}_{\eta, \tau}^{T} & 0 \\ 0 & \mathbb{B}_{\xi, \tau}^{T}\end{array}\right], \tilde{\mathbb{M}}_{\tau}:=$ $\left[\begin{array}{cc}\mathbb{M}_{\eta, \tau} & 0 \\ 0 & \mathbb{M}_{\xi, \tau}\end{array}\right], \tilde{\Xi}:=\left[\begin{array}{c}\mathbf{\Upsilon} \\ \boldsymbol{\Xi}\end{array}\right], \tilde{\mathbf{U}}:=\left[\begin{array}{l}\mathbf{U} \\ \mathbf{P}\end{array}\right]$ and $\tilde{\mathbf{F}}_{u}:=\left[\begin{array}{c}\mathbf{F}_{u} \\ 0\end{array}\right]$. 
The matrix that appear in the system of equations (33) can be factorized into an exact $L U$ matrix product as follows

$$
\begin{aligned}
\tilde{\mathbb{A}}:=\left[\begin{array}{cc}
\tilde{\mathbb{M}}_{\tau} & \tilde{\mathbb{B}}_{\tau}^{T} \\
\tilde{\mathbb{B}}_{\tau} & \tilde{\mathbb{K}}_{\tau}
\end{array}\right] & =\left[\begin{array}{cc}
\tilde{\mathbb{M}}_{\tau} & 0 \\
\tilde{\mathbb{B}}_{\tau} & \tilde{\mathbb{S}}
\end{array}\right]\left[\begin{array}{cc}
\mathbb{I} & \tilde{\mathbb{M}}_{\tau}^{-1} \tilde{\mathbb{B}}_{\tau}^{T} \\
0 & \mathbb{I}
\end{array}\right] \\
& =\left[\begin{array}{cc}
\mathbb{I} & 0 \\
\tilde{\mathbb{B}}_{\tau} \tilde{\mathbb{M}}_{\tau}^{-1} & \mathbb{I}
\end{array}\right]\left[\begin{array}{cc}
\tilde{\mathbb{M}}_{\tau} & \tilde{\mathbb{B}}_{\tau}^{T} \\
0 & \tilde{\mathbb{S}}
\end{array}\right],
\end{aligned}
$$

being $\tilde{\mathbb{S}}:=\tilde{\mathbb{K}}_{\tau}-\tilde{\mathbb{B}}_{\tau}^{T} \tilde{\mathbb{M}}_{\tau}^{-1} \tilde{\mathbb{B}}_{\tau}$ the Schur complement with respect to $\mathbf{U}$. In order to construct a preconditioner to the system (33) we build an inexact factorization of $\tilde{\mathbb{A}}$ such that each diagonal block is recursively preconditioned by another block preconditioner. We consider three different preconditioners of $\tilde{\mathbb{A}}$, which try to approximate the $L U$ decompositions (34)
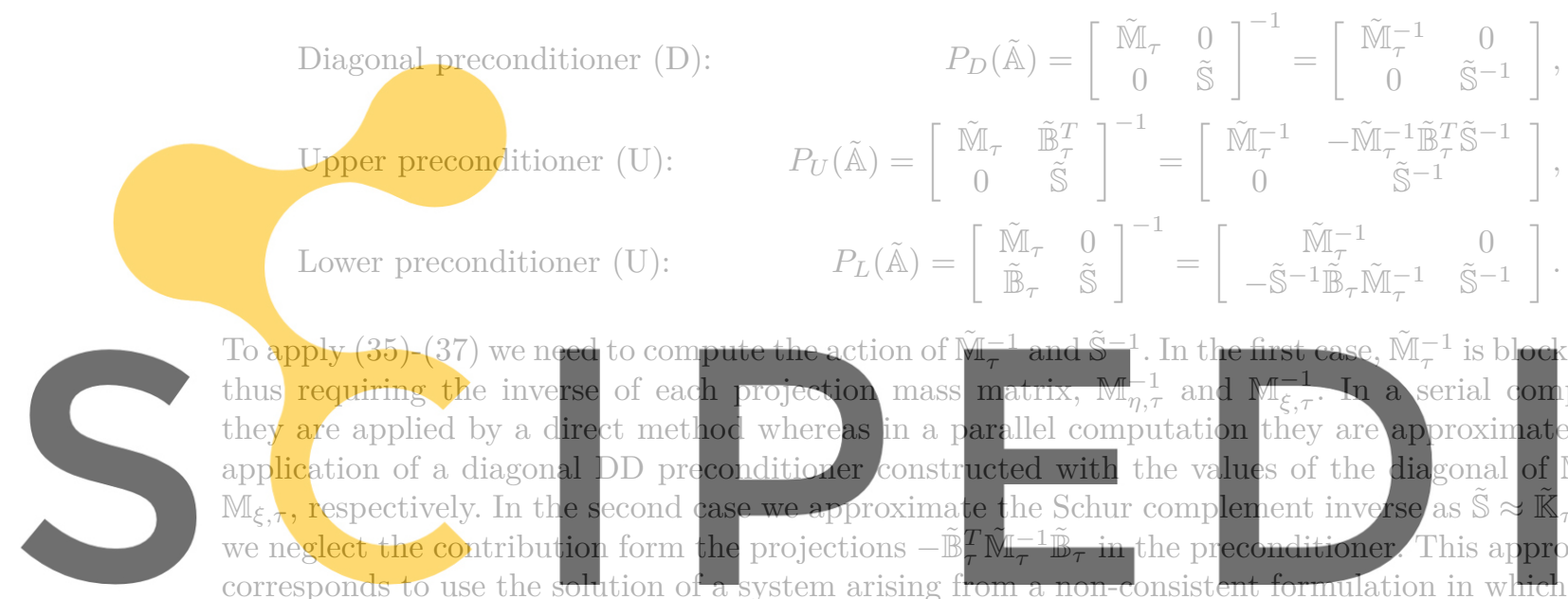

To apply (35)-(37) we neter thus requiring the inve they are applied by a dire application of a diagon $\mathbb{M}_{\xi, \tau}$, respectively. In the we neglect the contribution corresponds to use the
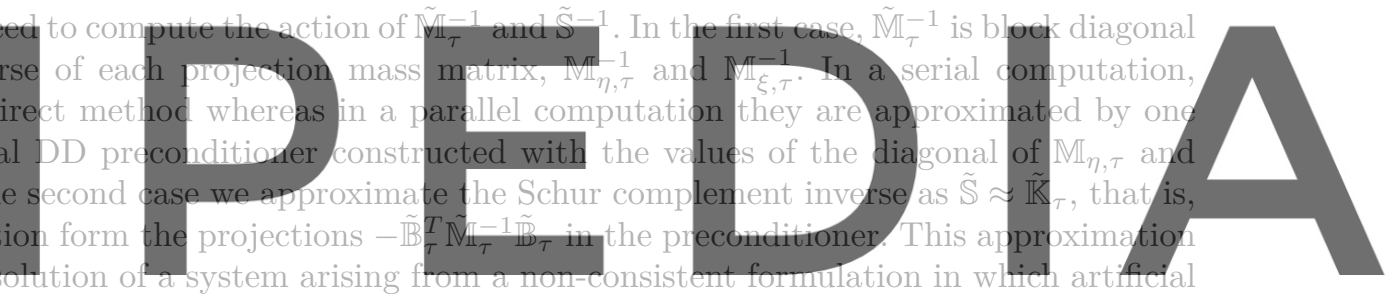
pressure and streamline diffusion are added. Stability of the preconditioner is therefore guaranteed.

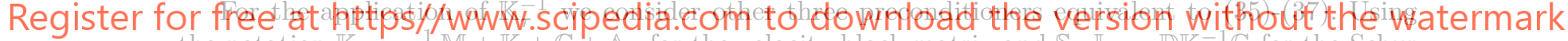

the notation $\mathbb{K}_{\tau}:=\frac{1}{\delta t} \mathbb{M}+\mathbb{K}+\mathbb{C}+\mathbb{A}_{\tau}$ for the velocity block matrix and $\mathbb{S}: \mathbb{L}_{\tau}-\mathbb{D}_{\tau}^{-1} \mathbb{G}$ for the Schur complement associated to the pressure field, we have the following block preconditioners

$$
\begin{array}{lr}
\text { Diagonal preconditioner (D): } & P_{D}\left(\tilde{\mathbb{K}}_{\tau}\right)=\left[\begin{array}{cc}
\mathbb{K}_{\tau} & 0 \\
0 & \mathbb{S}
\end{array}\right]^{-1}=\left[\begin{array}{cc}
\mathbb{K}_{\tau}^{-1} & 0 \\
0 & \mathbb{S}^{-1}
\end{array}\right], \\
\text { Upper preconditioner }(\mathrm{U}): & P_{U}\left(\tilde{\mathbb{K}}_{\tau}\right)=\left[\begin{array}{cc}
\mathbb{K}_{\tau} & \mathbb{G} \\
0 & \mathbb{S}
\end{array}\right]^{-1}=\left[\begin{array}{cc}
\mathbb{K}_{\tau}^{-1} & -\mathbb{K}_{\tau}^{-1} \mathbb{G S}^{-1} \\
0 & \mathbb{S}^{-1}
\end{array}\right], \\
\text { Lower preconditioner }(\mathrm{U}): & P_{L}\left(\tilde{\mathbb{K}}_{\tau}\right)=\left[\begin{array}{cc}
\mathbb{K}_{\tau} & 0 \\
\mathbb{D} & \mathbb{S}
\end{array}\right]^{-1}=\left[\begin{array}{cc}
\mathbb{K}_{\tau}^{-1} & 0 \\
-\mathbb{S}^{-1} \mathbb{D K}_{\tau}^{-1} & \mathbb{S}^{-1}
\end{array}\right] .
\end{array}
$$

As usual, the inverse of the Schur complement is approximated by a pressure Laplacian matrix, $\mathbb{S}^{-1} \sim \delta t \mathbb{L}_{p}^{-1}$. In regimes where the viscous term becomes dominant, one can use the Cahouet-Chabard preconditioner to approximate the inverse of the Schur complement, i.e., $\mathbb{S}^{-1} \sim\left(\nu+\tau_{c}\right) \mathbb{M}_{p}+\delta t \mathbb{L}_{p}^{-1}$. For the serial case, the Laplacian matrix is inverted using a direct method, but in a parallel context we approximate the inverse of $\mathbb{L}_{p}$ by one application of a BDDC preconditioner over such matrix. Something similar is done for the inverse of the velocity block matrix $\mathbb{K}_{\tau}$. Here we use a direct method for the serial case and one application of BDDC preconditioner when parallel solvers are treated. We refer to [33] for the application of the BDDC preconditioner to nonsymmetric problems. For a more detailed description of the implementation and algorithms used for the recursive block-preconditioning technique we refer to [25]. Besides monolithic approaches, splitting velocity-pressure techniques are commonly used for high Reynolds turbulent flows, which can be interpreted as one application of a block-preconditioner [34, 35]. High order time integration schemes that segregate velocity and pressure computation have recently been proposed in [36]. The use of the stabilized inf-sup stable FEs proposed herein are particularly well-suited for this type of schemes, because it keeps the index-2 differential-algebraic nature of the problem. 


\section{Numerical tests}

In this section we provide some numerical results for turbulent incompressible flow simulations using all the methods stated above. First, we do a comparative analysis between the three stabilization methods developed in Sect. 3. The parameter election for the pressure subscale term is assessed in all numerical tests performed in this section. But, first of all, we show some results about the computational cost of the VMS methods considered in this work. To check the efficiency of each method we solve a simple 2D steady problem with analytical solution.

\subsection{Analytical colliding flow}

In this section we solve a problem with analytical solution that models a colliding flow. This test have been used in [34] to discuss error estimates for the Stokes and Navier-Stokes problems using ISS FE velocity-pressure pairs. It has an analytical solution with the expression:

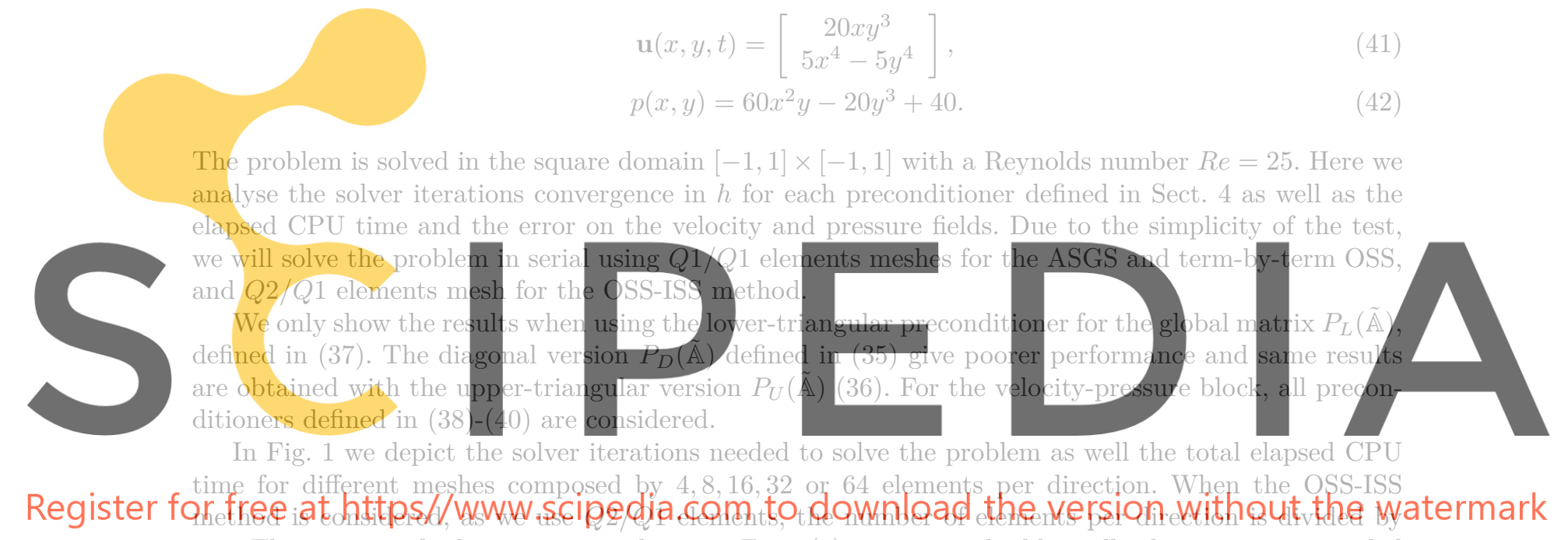

two. The amount of solver iterations shown in Fig. 1(a) is computed adding all solver iterations needed for all nonlinear iterations. We see in this figure that the OSS-ISS method has an increase of solver iterations for the coarser mesh, compared with the other two methods. This behaviour is produced by the fact that for this case the mesh is too coarse, $2 \times 2 Q 2 / Q 1$ elements, and the nonlinear iterations suffer a drastic increase. For finer meshes, the OSS-ISS method needs less solver iterations than the other two. We see that for the diagonal preconditioner $P_{D}\left(\mathbb{K}_{\tau}\right)$ the OSS-ISS is the only that scales when we refine the mesh. The upper and lower preconditioners, $P_{U}\left(\mathbb{K}_{\tau}\right)$ and $P_{L}\left(\mathbb{K}_{\tau}\right)$, have similar results with a slightly better performance of the upper version for all methods. The ASGS and OSS methods also need a similar number of iterations to solve the problem, being the OSS method a little bit over the ASGS.

Looking at the elapsed computational time, Fig. 1(b), we see that the OSS method is clearly more expensive, in terms of consumed time, than the other two methods. The ASGS method is slightly faster than the OSS-ISS, but the differences are almost negligible for the $P_{U}\left(\mathbb{K}_{\tau}\right)$ preconditioner. The fact that the most expensive method is the OSS method is justified by the number of Degrees Of Freedom (DOFs) that appear in the system of equations. As stated in Sect. 4, the OSS method has two vectorial unknowns more than ASGS method. On the other hand, the OSS-ISS method also has an additional vectorial unknown than ASGS, but the pressure field is approximated with half nodes. Without taking into account the boundary conditions, for the $4 \times 4$ elements mesh $(2 \times 2$ for the OSS-ISS case), we would have 175 DOFs for the OSS method, 75 DOFs for the ASGS method and 109 DOFs for the OSS-ISS method. Then, we see that, although it has more DOFs, the OSS-ISS method is comparable in terms of computational time with the ASGS. Further, the number of DOFs is a wrong measure of the CPU cost. The most expensive problem in such simulations is the pressure Poisson equation, which in this case is smaller (one order less) for ISS elements. Further, the projection DOFs are almost for free, since they just involve a mass matrix solve. Further, the ISS method exhibit a clearly lower number of iterations. 


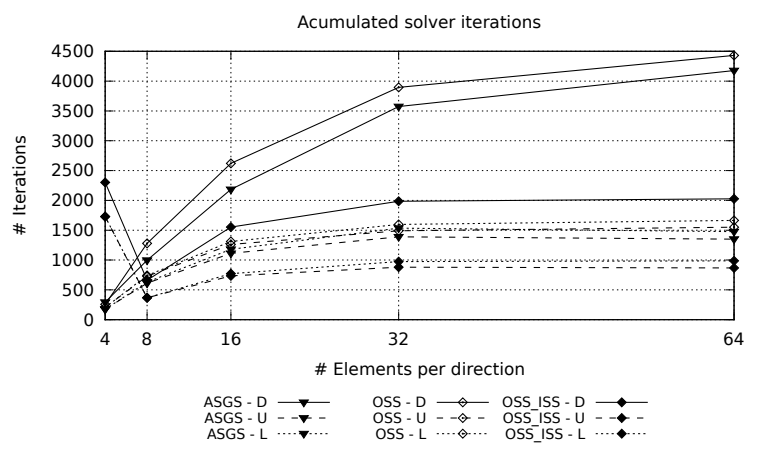

(a) Solver iterations

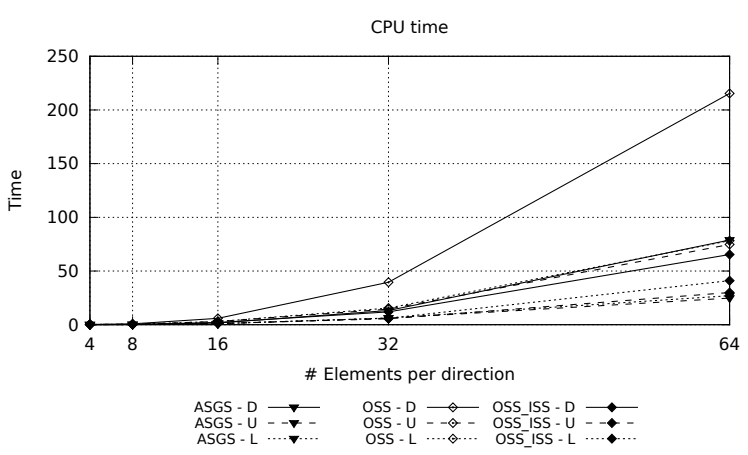

(b) Elapsed CPU time

Figure 1: Colliding flow solver iterations and elapsed CPU time using $P_{U}(\tilde{\mathbb{A}})$ for the global matrix.

Let us now focus on the error in the velocity and pressure fields. Since we are solving a problem with analytical solution, we can evaluate exactly the error of the FE approximation, $e_{u}:=\left\|\mathbf{u}_{h}-\mathbf{u}\right\|$ and $e_{p}:=\left\|p_{h}-p\right\|$. Fig. 2 depicts the convergence of both errors when refining the mesh. In Fig. 2(a) we see that the velocity error converges as expected, with a 2nd order rate for the ASGS and OSS methods, which are approximated by $Q 1 / Q 1$ elements, and with a 3rd order rate for the OSS-ISS method, which is approximated by a $Q 2 / Q 1$ element. Looking at Fig. 2(b), it is seen that the pressure

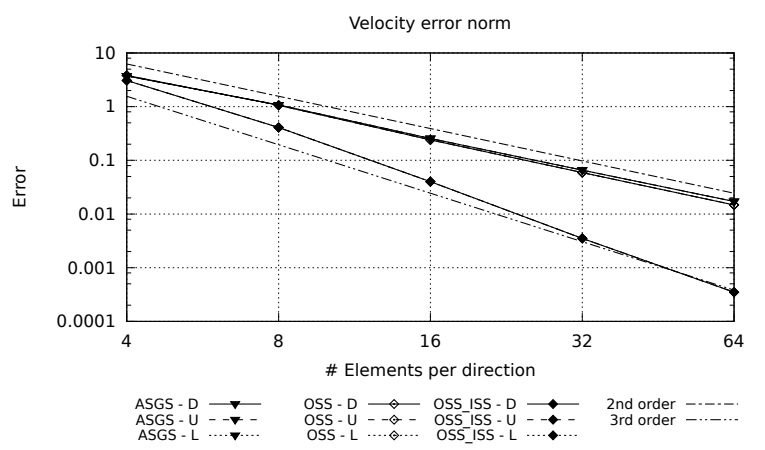

(a) Velocity error

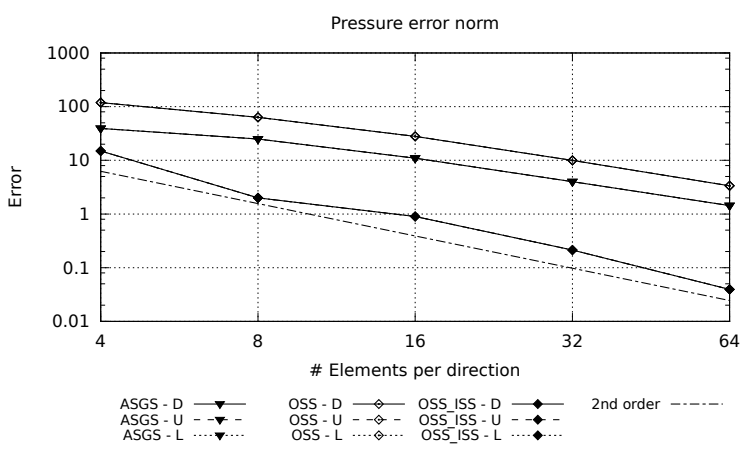

(b) Pressure error

Figure 2: Colliding flow error convergence using $P_{U}(\tilde{\mathbb{A}})$ for the global matrix.

error norm converges with a 2nd order rate for all the methods, as expected. Note that there is no difference between the results changing the preconditioner, since the solution is the same for all cases because we are not modifying the system that is solved.

In order to check the efficiency of each method we compare the error norm with the elapsed computational time. In this way we have an idea of the time needed for a given method to achieve certain solution accuracy. Fig. 3 shows this comparison for velocity (Fig. 3(a)) and pressure (Fig. 3(b)) fields, where we see that, excluding the coarser mesh, the OSS-ISS method is much more efficient than the other two. Furthermore, the most efficient preconditioner is the upper version $P_{U}\left(\mathbb{K}_{\tau}\right)$.

\subsection{Taylor Green Vortex flow}

It has been shown by many authors that VMS stabilization terms can act as a LES model for turbulent flows, introducing the appropriate dissipation of the small scales that are not captured by the coarse solution. In particular, ASGS and OSS methods were assessed in [16], showing that these methods are capable to perform a good LES simulation of different turbulent benchmark problems. In order to check the performance of the convection-only OSS stabilization of ISS elements for a LES 


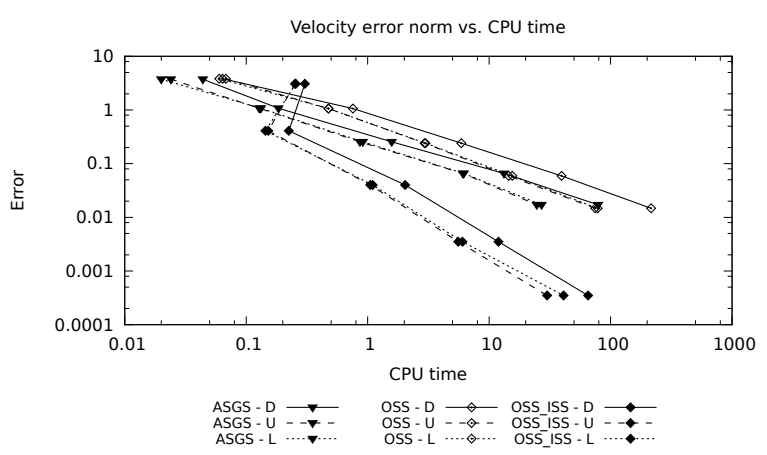

(a) Velocity error vs. CPU time

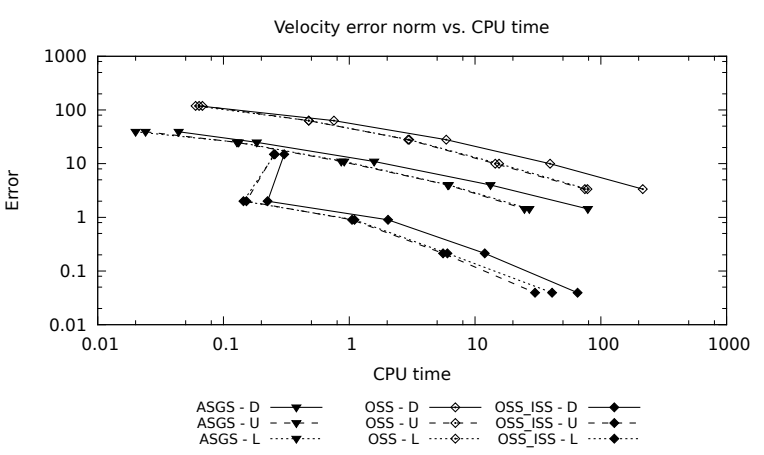

(b) Pressure error vs. CPU time

Figure 3: Colliding flow error vs. CPU time using $P_{U}(\tilde{\mathbb{A}})$ for the global matrix.

simulation, we analyse its behaviour in the Taylor Green Vortex (TGV) flow. This problem is widely used for benchmarking turbulent numerical simulations.

\subsubsection{Test setting}

The computational domain is the cube $(0,2 \pi)^{3}$ with periodical boundary conditions and the initial condition is given by an analytical field (see, e.g., [37])

$$
\begin{aligned}
& \mathbf{u}(x, y, z, 0)=\left(\begin{array}{l}
u_{x} \\
u_{y} \\
u_{z}
\end{array}\right)=\left(\begin{array}{c}
u_{0} \cos (x) \sin (y) \sin (z) \\
-u_{0} \sin (x) \cos (y) \sin (z) \\
0
\end{array}\right) \\
& p(x, y, z, 0)=p_{0}+\frac{1}{16}(\cos (2 x)+\cos (2 y))(\cos (2 z)+2),
\end{aligned}
$$

with

$$
u_{0}=\frac{2}{\sqrt{3}} \sin \left(\gamma+\frac{2 \pi}{3}\right)
$$

We choose $\gamma=0$, which gives the mean initial velocity $u_{0}=1$. We solve the TGV problem using a Reynolds number $\operatorname{Re}=1600$.

\subsubsection{Comparison between different VMS methods}

The problem is solved from $t=0.0$ to $T=10.0$ with a fixed time step size of $\delta t=5.0 \cdot 10^{-2}$ using a Crank-Nicolson time integration scheme, and the results are compared against a DNS by Brachet et al. [37]. We discretize the domain using different choices of the number of elements and the order of approximation, having two differentiated group of discretizations; one with $32^{3}$ velocity DOFs and another with $64^{3}$ velocity DOFs. The former will be composed by the following meshes: $32^{3} Q 1 / Q 1,16^{3}$ $Q 2 / Q 2$ elements or $16^{3} Q 2 / Q 1$ elements when we use ISS discretization. The second group of meshes is made by: $64^{3} Q 1 / Q 1,32^{3} Q 2 / Q 2$ or $32^{3} Q 2 / Q 1$ elements. For the stabilized formulations, ASGS and OSS, the algorithmic constants are $c_{1}=12 c_{2}=2$ and $c_{c}=0.0$, and for the OSS-ISS method the same $c_{1}$ and $c_{2}$ algorithmic constants are used, but $c_{c}=4.0$ unless noted otherwise. This choice of $c_{c}$ for the OSS-ISS method is assessed in a following subsection.

In Fig. 4 we show the energy evolution and the energy dissipation rate for the ASGS, the OSS, and the OSS-ISS methods. A first thing that we have to state at this point is that the ASGS method with $32^{3} Q 1 / Q 1$ has failed to converge at early stages of the problem, a behaviour also observed in [16]. Looking at Fig. 4(a) it is clear that the degree of interpolation makes a great difference on the solution, even with the same number of DOFs, the solution is more accurate when a higher order of interpolation is used. In the same figure we see that the loose of precision in the pressure for the ISS elements $Q 2 / Q 1$ does affect the solution, giving a result between the $Q 1 / Q 1$ and $Q 2 / Q 2$ solutions. At Fig. 4(b) we see that the OSS-ISS method is more dissipative than the others. This behaviour is caused by the lack of 


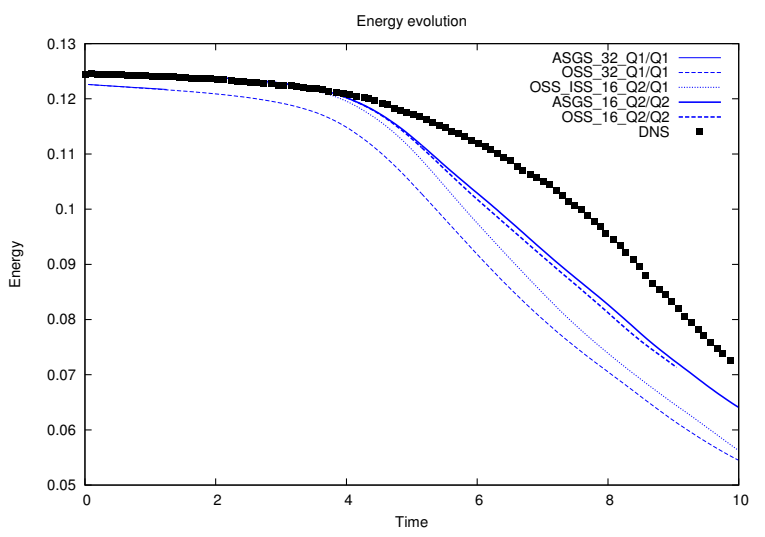

(a) Energy evolution

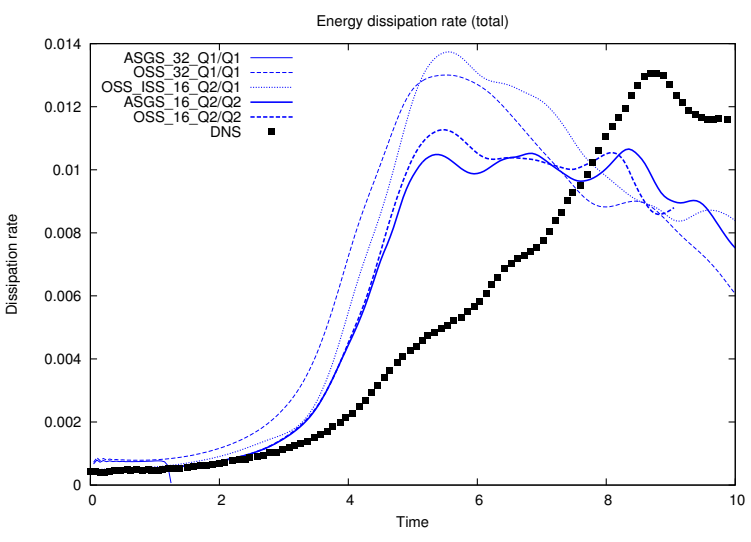

(b) Total energy dissipation rate

Figure 4: Energy and Total energy dissipation rate evolution with $32^{3}$ velocity DOFs

accuracy in the pressure field, which in turn affects the conservation of mass of the problem. A more exhaustive analysis of the effect of this term is done below.

Although an important improvement of the solution is achieved by increasing the order of interpolation, $32^{3}$ velocity DOFs is still a very coarse mesh and the results shown in Fig. 4 are far from the DNS ones. Then, the same problem is solved in a finer mesh, with the double of velocity DOFs per direction, with the results shown in Fig. 5. In this case, all methods converge at all time steps. Fig. 5(a) depicts the energy evolution and it is also seen that the increase on the degree of interpolation results in a more accurate solution. Both ASGS and OSS methods with $32^{3} Q 2 / Q 2$ elements have very accurate results, providing a solution almost on top of the DNS. There are very little differences between stabilization methods when the same discretization is used. Furthermore, the results of the OSS-ISS method are closer to the $Q 1 / Q 1$ discretization than to the $Q 2 / Q 2$ one. The total energy dissipation rate shown in Fig. 5(b) denote a very good agreement of the $Q 2 / Q 2$ solution with the DNS, while the $Q 1 / Q 1$ discretization for both ASGS and OSS methods are still more diffusive. Note that the OSS-ISS method for this discretization has more or less the same energy dissipation than the $Q 1 / Q 1$ discretizations.

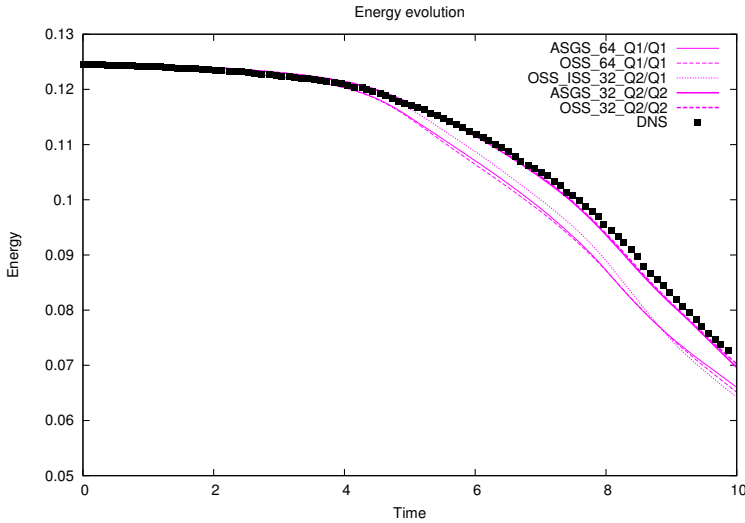

(a) Energy evolution

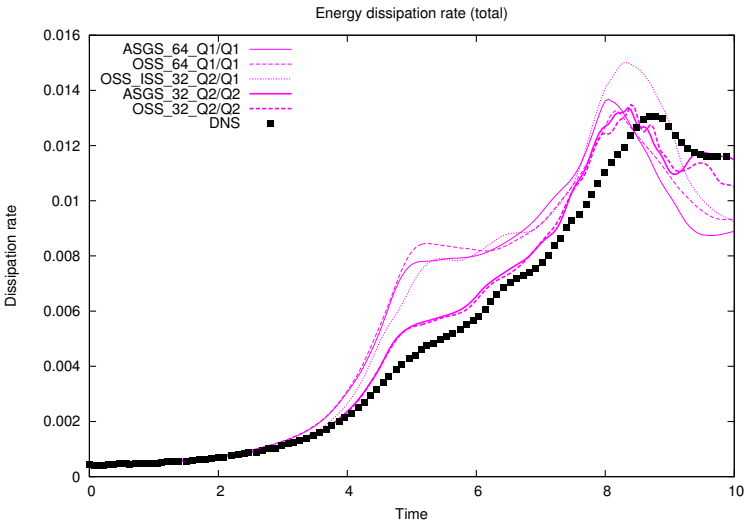

(b) Total energy dissipation rate

Figure 5: Energy and Total energy dissipation rate evolution with $64^{3}$ velocity DOFs

When analysing the suitability of a LES model, a very important turbulent quantity to take into account is the energy spectra. It gives us information about how the energy is distributed among the scales of the problem. In order to assess the behaviour of the proposed methods in that aspect, we compare our results by the DNS by Gassner et al. [38]. In Fig. 6 the energy spectra at $t=9.0$ is 
depicted for both discretization groups. The coarser cases shown in Fig. 6(a) are all far from the DNS result, but follow the same pattern, with most of the energy on the greatest scales and little energy on the small scales, without any pileup of energy on the small scales. When the mesh is refined, see Fig. 6(b), the computed energy spectra tends to the DNS one. Note that also in this plot, $Q 2 / Q 2$ discretization have better agreement with the DNS, specially on the small scales, where the influence of the enrichment of the interpolation space is patent.

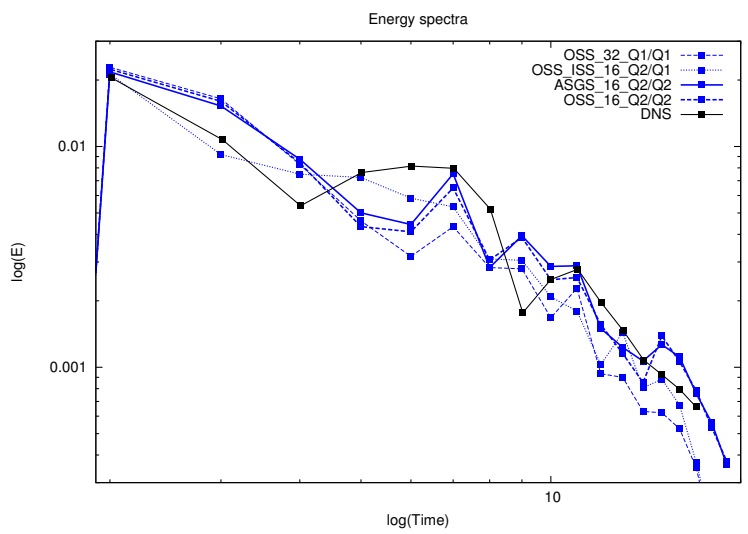

(a) $32^{3}$ velocity DOFs

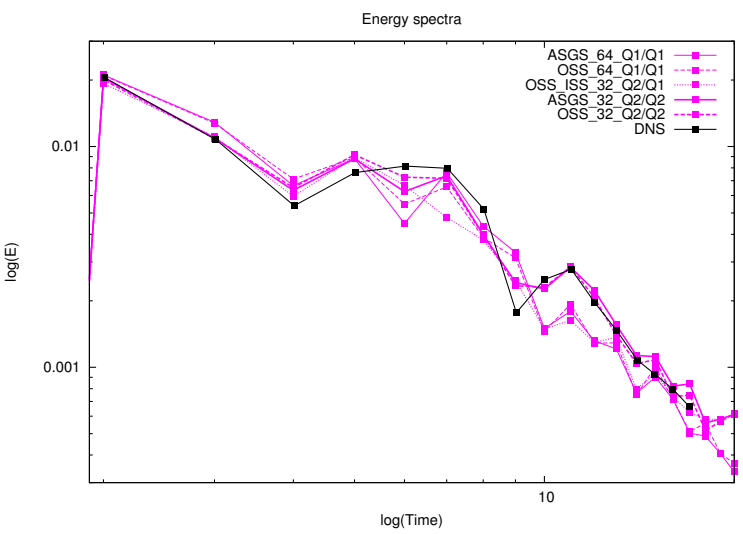

(b) $64^{3}$ velocity DOFs

Figure 6: Energy spectra at $t=9.0$

\subsubsection{Computational cost}

It is clear that a good LES model has to reproduce as accurately as possible all the turbulent quantities, even for coarse meshes. The results presented until now show that ASGS and OSS methods perform better than the OSS-ISS when $Q 2 / Q 2$ elements are used. Additionally, it has been seen that all methods converge to the DNS results when the mesh is refined. But a crucial point that has to be always taken into account when we talk about numerical simulations is the computational cost. At the end, we are looking for the cheapest method that allow us to reproduce accurately the physical phenomena that takes place in a turbulent flow. So in this subsection we will discuss the computational cost associated to each method and their efficiency when solving this kind of flows.

To check the computational cost we look at the number of solver iterations needed for each method, shown in Fig. 7. In particular, Fig. 7(a) depicts the total amount of solver iterations at each time step, adding up all nonlinear iterations. Note that the change of the nonlinear iterations along the time can be clearly noticed by the jumps on the curves. We see that the cheapest method is the OSS-ISS for both discretizations, $32^{3}$ and $64^{3}$ velocity DOFs, with much less solver iterations per time step than the other methods. It is also seen that ASGS is a little bit cheaper than the OSS method, which can be caused by the size of the system, much bigger for the OSS case due to the implicit treatment of the projections. An interesting result also seen in $7(\mathrm{a})$ is the improvement on the computational cost when we go from $Q 1 / Q 1$ to $Q 2 / Q 2$ discretization, keeping constant the number of DOFs. Looking at 7 (b) we realize that at the end of the computation, the total amount of solver iterations needed by OSS-ISS method is around 1.5 times less than the ASGS method, for the finer mesh, and half of them for the coarse mesh. This result indicates that the OSS-ISS is a very good approach as a LES model for turbulent problems since, although it has been seen that is not the most accurate when looking to the turbulent quantities, it is much cheaper and we can refine the mesh in order to get better results remaining competitive with the other methods. Further, let us remark that the pressure Poisson solvers are the most computationally intensive, and ISS methods involve Q1 pressure spaces for Q2 ones for stabilized methods.

\subsubsection{Influence of the pressure subscale term}

As said above and also stated in Sect. 3.4, the pressure subscale $\tau_{c}\left(\nabla \cdot \mathbf{u}_{h}, \nabla \cdot \mathbf{v}_{h}\right)$ term has an important role when using ISS elements. In this subsection we are going to analyse the effect of this 


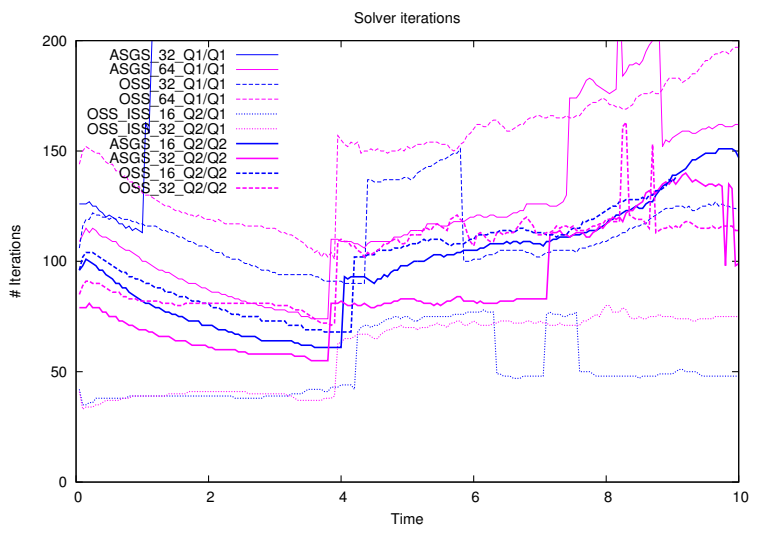

(a) Solver iterations per time step

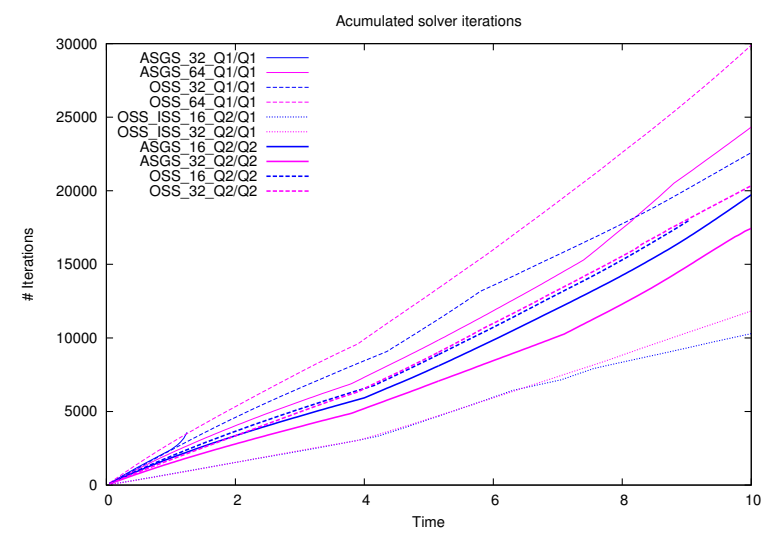

(b) Acumulated solver iterations

Figure 7: Computational cost

term on the results when simulating the TGV problem. Hence, we redefine the $\tau_{c}$ definition in (17) by

$$
\tau_{c}=c_{c}\left(\nu+\frac{c_{2}}{c_{1}} h\left|\mathbf{u}_{h}\right|\right),
$$

which is equivalent to (17) when $c_{c}=1.0$. We keep the algorithmic parameters $c 1=12.0$ and $c 2=2.0$ constant, so considering different values of $c_{c}$ we can evaluate the influence of the pressure subscale term on the solution. In this case we choose six different configurations $c_{c}=\{0.0,0.25,0.5,1.0,2.0,4.0\}$ and solve the problem with the $16^{3} Q 2 / Q 1$ elements mesh. We compare the solution against the one obtained with the OSS method with $16^{3} Q 2 / Q 2$ elements discretization.

In Fig. 8(a) the energy dissipation rate of the FE counterpart is shown. That is the viscous term $\nu\left\|\nabla \mathbf{u}_{h}\right\|^{2}$ that appears in equation (31). We see that when we reduce $c_{c}$ the method becomes more dissipative and it may seem a good idea to keep this term as small as possible in order to get closer to the DNS curve. But if we look at the energy spectra shown in Fig. 8(b), we see that this is not a good choice. What is actually happening when $c_{c}$ goes to zero is that the dissipation is taking place in the largest scales of the problem, while the smallest ones keep the energy, resulting in an energy pileup at the tail of the spectra. This means that the energy is not dissipating in the correct way and the small scales, which are the ones that have more influence on the viscous dissipation term, have more energy than the desired one. Therefore, a good selection is to choose $c_{c}=4.0$, which results are closer to the OSS method and has a better energy spectra shape. A higher value of $c_{c}$ eventually lead to unstable solutions, for instance, with $c_{c}=8.0$ and $\delta t=5.0 \cdot 10^{-2}$ the solution fails to converge at $t=0.4$.

As stated in Sect. 3.4, the pressure subscale term is essential to enforce the incompressibility constrain at the discrete level. In Fig. 9 the total energy dissipation rate (Fig. 9(a)) is depicted together with the velocity divergence $L 2$-norm $\left\|\nabla \cdot \mathbf{u}_{h}\right\|$ (Fig. 9(b)). We see that, effectively, $\left\|\nabla \cdot \mathbf{u}_{h}\right\|$ is reduced when $\tau_{c}$ is increased. The case of $c_{c}=0.0$ give especially bad results in terms of mass conservation, affecting also to the energy dissipation rate. In this case, as seen in Fig. 8(b), the over-dissipation on the large scales and the under-dissipation on the smallest ones makes the spatial derivatives too relevant on the fluid flow, increasing the $\left\|\nabla \cdot \mathbf{u}_{h}\right\|$ term. The introduction of $\tau_{c}\left\|\nabla \cdot \mathbf{u}_{h}\right\|$ into the energy dissipation equation (31) changes the way in which the flow dissipates its energy among the different scales, decreasing the energy of the small scales and, then, reducing the importance of the spatial derivatives, which is reflected in a lower value of $\left\|\nabla \cdot \mathbf{u}_{h}\right\|$ but also in a lower dissipation rate, as seen in Fig. 9(a), even if a positive term has been added to (31).

\subsubsection{Refinement analysis for the OSS-ISS method}

In the computational cost analysis shown above we have seen that OSS-ISS has a great potential as a LES model, especially when we the computational cost is taken into account. This subsection aims 


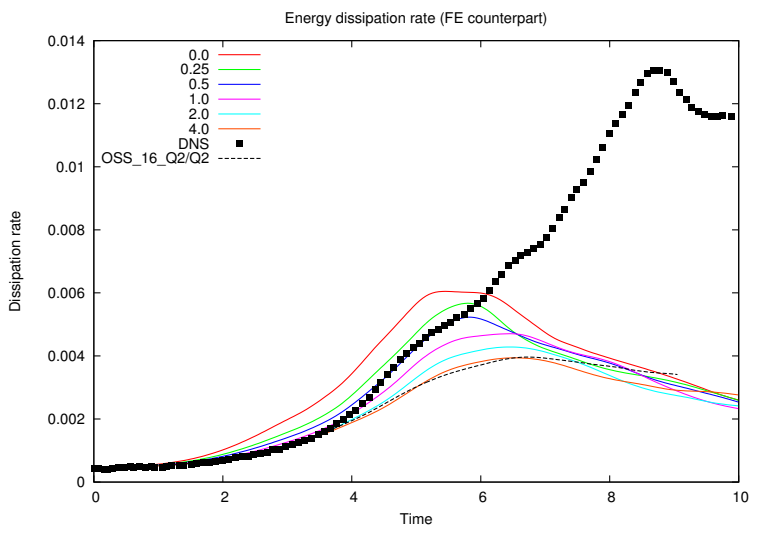

(a) Viscous energy dissipation rate

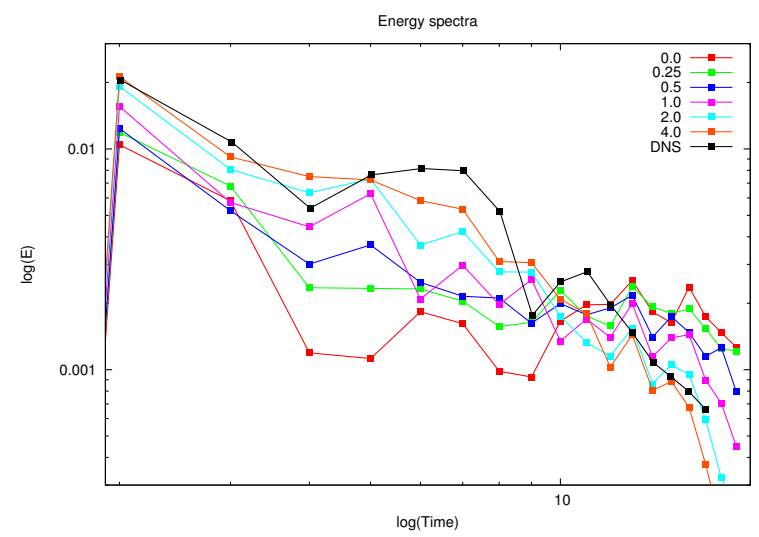

(b) Energy spectra

Figure 8: Comparison for different $c_{c}$ choices for OSS-ISS with $32^{3}$ velocity DOFs

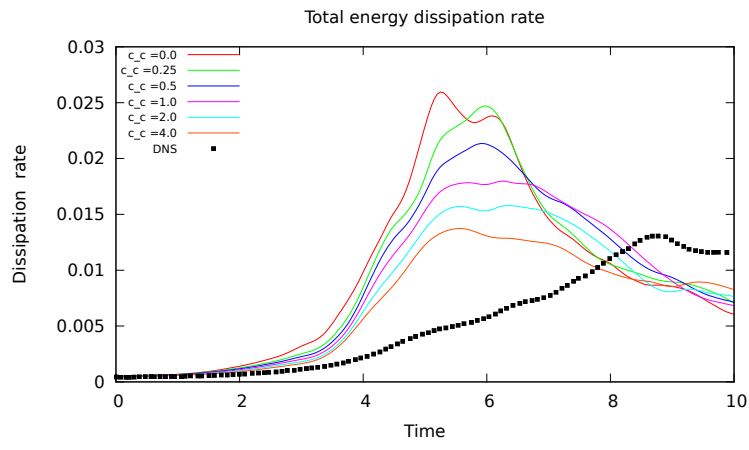

(a) Total energy dissipation rate

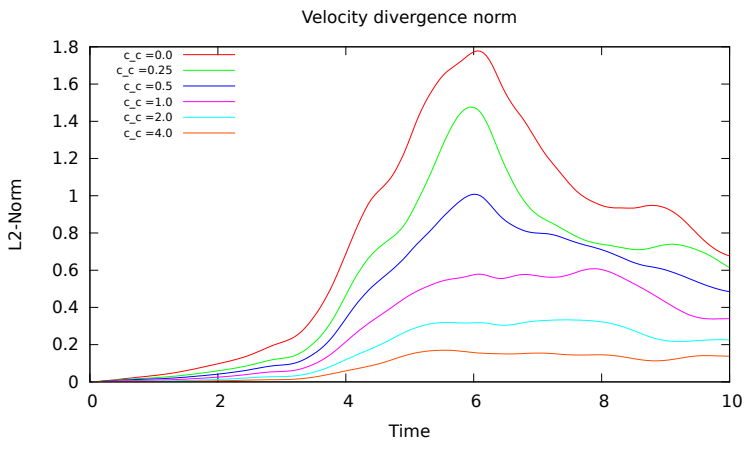

(b) $\left\|\nabla \cdot \mathbf{u}_{h}\right\|$

Figure 9: Total energy dissipation and velocity divergence $L 2$-norm for different $c_{c}$ choices for OSS-ISS with $32^{3}$ velocity DOFs

to check the performance of this method when we refine the mesh both reducing the element size and increasing the interpolation order.

Once determined that the best algorithmic constant for the pressure subscale term is $c_{c}=4.0$ when ISS FEs are used, we keep this value constant and change the discretization. A refinement analysis can be done to determine if the LES approach presented in this work effectively converge to the DNS solution when we refine the mesh. In section 5.2 the TGV problem has been solved using different discretizations with $32^{3}$ and $64^{3}$ velocity DOFs. Now we go further and also solve the problem with $96^{3}$ velocity DOFs, that is a $48^{3} Q 2 / Q 1$ elements mesh. Furthermore, here we also use $Q 3 / Q 2$ FEs. In particular, a $21^{3}$ and $32^{3} Q 3 / Q 2$ elements meshes are used, corresponding to the group of $64^{3}$ and $96^{3}$ velocity DOFs, respectively. We also decrease the time step to $\delta t=2.5 \cdot 10^{-2}$ for the discretizations with $64^{3}$ velocity DOFs and $\delta t=1.5 \cdot 10^{-2}$ for the discretizations with $96^{3}$ velocity DOFs.

In Fig. 10 we depict the kinetic energy and the total energy dissipation rate evolution for the different discretizations considered in this refinement analysis. Looking at the energy evolution in Fig. 10(a) it is clearly seen that the solution converge to the DNS results, giving the $32^{3} Q 3 / Q 2$ elements mesh a very accurate solution, which is also evident in Fig. 10(b) where the total energy dissipation rate of this discretization is on top of the DNS solution. We also see in Fig. 10 the relevance of the degree of interpolation, giving much better results the discretization of higher order for a given number of DOFs.

Now, we ask ourselves how the tuning of the parameter $\tau_{c}$ affects when finer meshes are used. In particular, we want to know how important the $c_{c}$ parameter becomes when higher order FEs are used. 


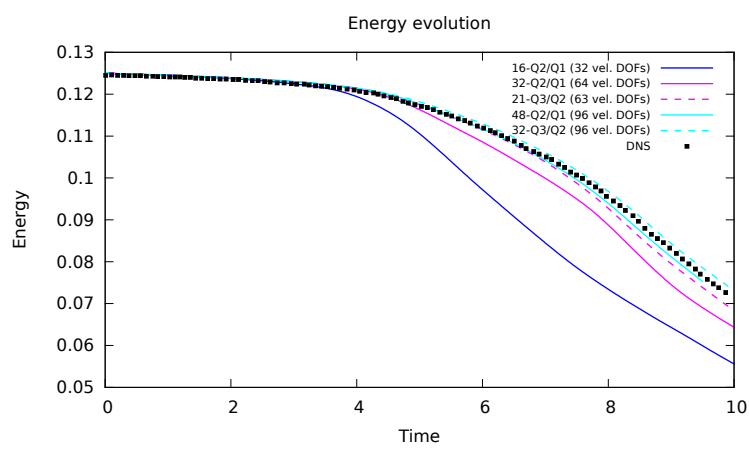

(a) Energy evolution

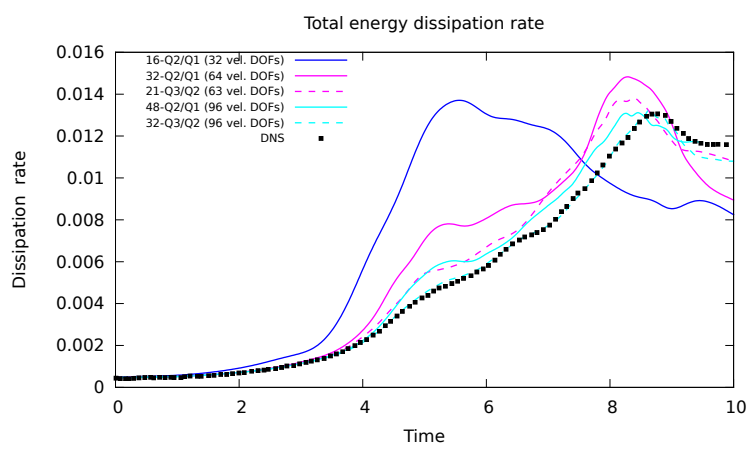

(b) Total energy dissipation rate

Figure 10: Energy and Total energy dissipation rate evolution refining the mesh with $c_{c}=4.0$.

To answer this question we solve the TGV problem for two different discretizations: $32^{3} Q 2 / Q 1$ and $21^{3}$ Q3/Q2 elements meshes, and three different values of $c_{c}: 0.0,1.0$ and 4.0.

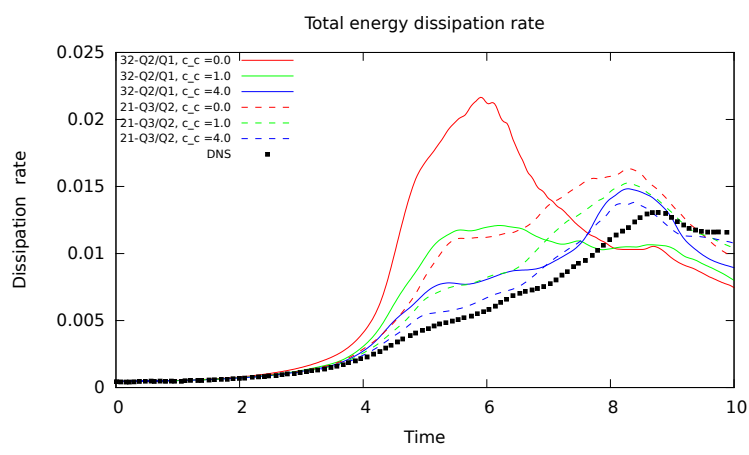

(a) Total energy dissipation rate

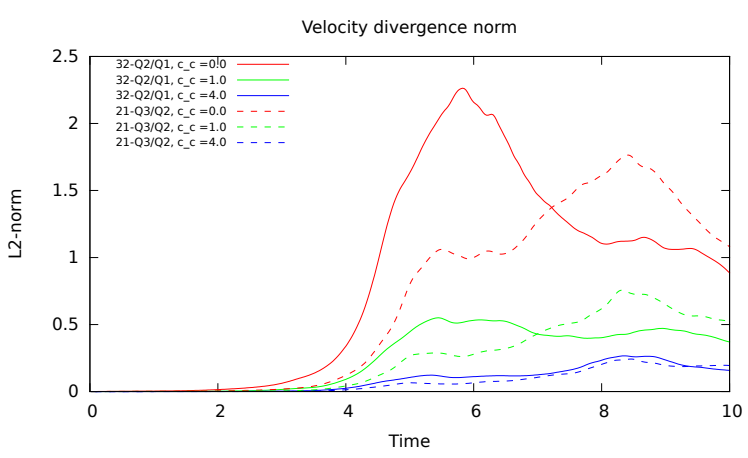

(b) $\left\|\nabla \cdot \mathbf{u}_{h}\right\|$

Figure 11: Total energy dissipation and velocity divergence $L 2$-norm for different $c_{c}$ choices for OSS-ISS with $64^{3}$ velocity DOFs

Fig. 11 depicts the total energy dissipation rate (Fig. 11(a)) and the velocity divergence L2-norm (Fig. 11(b)) for the two discretizations considered and for different choices of $c_{c}$. We still see a dependence on $c_{c}$, but differences reduce when higher order of interpolation is used, as expected. It is also seen that when increasing $c_{c}$, the differences between the two discretizations are reduced (for a fixed $c_{c}$ ). This behaviour is particularly significant when we look at the velocity divergence norm in Fig. 11(b), where we see that for $c_{c}=0$ the two discretizations give a completely different evolution of $\left\|\nabla \cdot \mathbf{u}_{h}\right\|$, while for $c_{c}=4.0$ the results are almost the same. In the same figure, we can see that the largest value of $\left\|\nabla \cdot \mathbf{u}_{h}\right\|$ for $c_{c}$ is larger than the largest one depicted in Fig. 9(b), which is a result of a $16^{3} Q 2 / Q 1$ elements mesh.

Going further we also do the same test for the $48^{3} Q 2 / Q 1$ and $32^{3} Q 3 / Q 2$ elements mesh (Fig. 12). In this figure we see that the differences between the three $c_{c}$ cases are reduced. Looking at Fig. 12(a) it is seen that for $c_{c}=0$ and $Q 2 / Q 1$ elements, although the result is far from the DNS, the maximum value of the dissipation rate is much lower than the given in Fig. 11(a). When using $Q 3 / Q 2$ elements, the changes on $c_{c}$ produce lower differences compared against the $Q 2 / Q 1$ approximation. The divergence norm depicted in Fig. 12 also show improvements with respect to Fig. 11. In this case, the maximum value of the divergence for $c_{c}$ for $Q 2 / Q 1$ elements is lower than the case of $64^{3}$ velocity DOFs and also for the case of $32^{3}$ velocity DOFs.

To summarize, we have checked that refining the mesh we converge to the DNS results and, for a given number of DOFs, the results improve when higher order interpolation is used. On the hand, we 


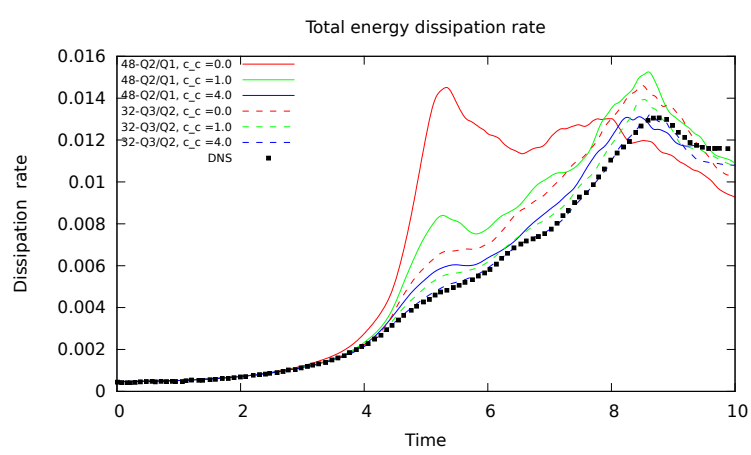

(a) Total energy dissipation rate

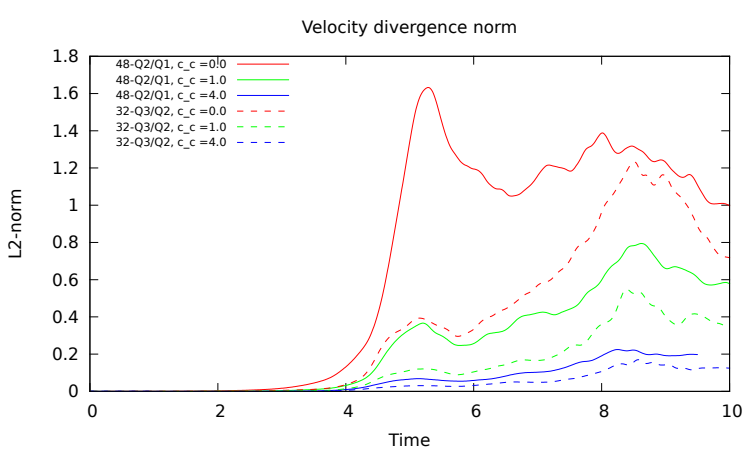

(b) $\left\|\nabla \cdot \mathbf{u}_{h}\right\|$

Figure 12: Total energy dissipation and velocity divergence $L 2$-norm for different $c_{c}$ choices for OSS-ISS with $96^{3}$ velocity DOFs

have seen that increasing $c_{c}$ we reduce $\|\nabla \cdot \mathbf{u}\|$, and consequently the accuracy of the solution.

\subsection{Turbulent channel flow at $R e_{\tau}=395$}

In Sect. 5.2 we have tested an homogeneous turbulent flow. Now, we want to check the behaviour of the proposed OSS-ISS stabilization method for a wall bounded turbulent test. To do so, we use the Turbulent Channel Flow (TCF) test with a Reynolds number based on the wall friction $\left(R e_{\tau}\right)$ equal to 395. This test was exhaustively tested using a VMS method with OSS in [16].

\subsubsection{Test setting}

The domain of the TCF problem for $R e_{\tau}=395$ is given by a box of length $(2 \pi \delta \times 2 \delta \times 2 / 3 \pi \delta)$. The $x$-direction is the flow direction, also called stream-wise direction, the $y$-direction is the wall-normal direction, and the $z$-direction is the span-wise direction. Homogeneous Dirichlet boundary conditions for the velocity DOFs are imposed on wall-normal direction boundaries $(y=-\delta$ and $y=\delta)$, while periodic boundary conditions are defined on the stream-wise and span-wise directions. Fig. 13 depicts schematically the geometry of the computational domain for this test.

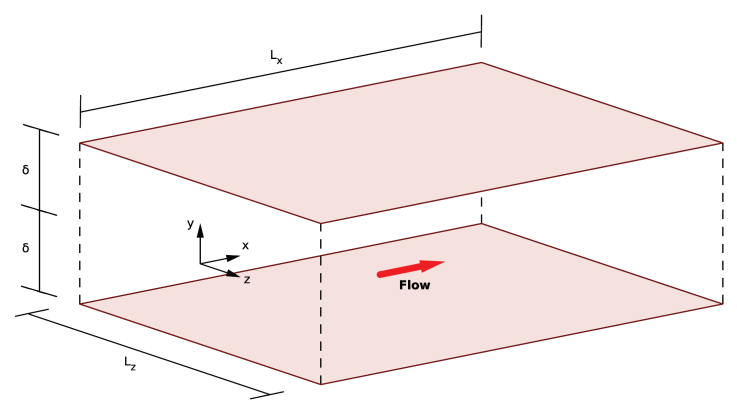

Figure 13: Channel computational domain.

The problem is solved using two different meshes, with $32^{3} Q 2-Q 1$ and $21^{3} Q 3 / Q 2$ elements mesh. Both meshes have refined elements near the wall in the wall-normal direction, like the one used in [16]. The algorithmic constants that appear in the OSS-ISS method will be discussed in the following subsections.

The obtained results are compared against a DNS computed in [39, 40] (MKM-DNS), then, the parameter election will be according to the ones defined in the cited paper. The bulk mean velocity and the half channel height are taken equal to one, $\bar{U}=1$ and $\delta=1$. Knowing the estimated Reynolds number based on the bulk mean velocity, $R e=\bar{U} 2 \delta / \nu \approx 13,750$ (see [41]), one can obtain the value 
of the viscosity, $\nu=1.4545 \cdot 10^{-4}$. From the Reynolds number based on the friction velocity, we can determine the friction velocity magnitude: $u_{\tau}=R e_{\tau} \nu / \delta=5.745 \cdot 10^{-2}$. Thus, the wall shear stress reads $\tau_{w}=u_{\tau}^{2}=3.3010 \cdot 10^{-3}$. A force equivalent to a pressure gradient is imposed to drive the movement of the flow in the stream-wise direction, $f_{x}=\tau_{w} / \delta$.

In order to achieve the statistically steady state solution, an initial solution is provided following [42]. This initial solution consists in a unidirectional velocity profile over which is added a fluctuation:

$$
\begin{aligned}
& u_{x}=C\left(1-y^{8}\right)+\epsilon \frac{L_{x}}{2} \sin (\pi y) \cos \left(\frac{4 \pi x}{L_{x}}\right) \sin \left(\frac{2 \pi z}{L_{z}}\right), \\
& u_{y}=-\epsilon(1+\cos (\pi y)) \sin (\pi y) \sin \left(\frac{4 \pi x}{L_{x}}\right) \sin \left(\frac{2 \pi z}{L_{z}}\right), \\
& u_{z}=-\epsilon \frac{L_{z}}{2} \sin \left(\frac{4 \pi x}{L_{x}}\right) \sin (\pi y) \cos \left(\frac{2 \pi z}{L_{z}}\right) .
\end{aligned}
$$

The constant $C$ is chosen in a such way that the field without fluctuations would have a bulk mean velocity $\bar{U}=1.0$. The fluctuation constant $\epsilon$ is $10 \%$ of the bulk mean velocity.

\subsubsection{Effect of the pressure subscale term on the conservation of mass}

As it has been said in Sect. 3.4, the pressure subscale term has a noticeable effect on the solution when ISS elements are used. The effect of this term has also been analysed in a previous test, see Sect. 5.2. Here we will also assess the effect of this term on a wall-bounded flow.

First we will focus on the effect of the second term in (44), looking how the $c_{2} / c_{1}$ ratio affects the solution keeping $c_{c}=1.0$. As we are in a turbulent regime, we do not expect that the viscous counterpart in 16 will have relevance on the solution, but we do expect it for the convective counterpart. Then, we will keep $c_{1}=12.0$ and we will increase $c_{2}$ from 1.0 to 16.0. The variations on $c_{2}$ not only have an effect on $\tau_{c}$ but also on $\tau_{m}$. We solve the problem from $t=0$ to $t=20 \pi$ (time needed to cross the channel 10 times, based on the initial mean bulk velocity $\bar{U}$ ) starting from the initial solution (45) using the implicit version of (3-3) SRK scheme. The energy and $\left\|\nabla \cdot \mathbf{u}_{h}\right\|$ evolution are plotted in Fig. 14.

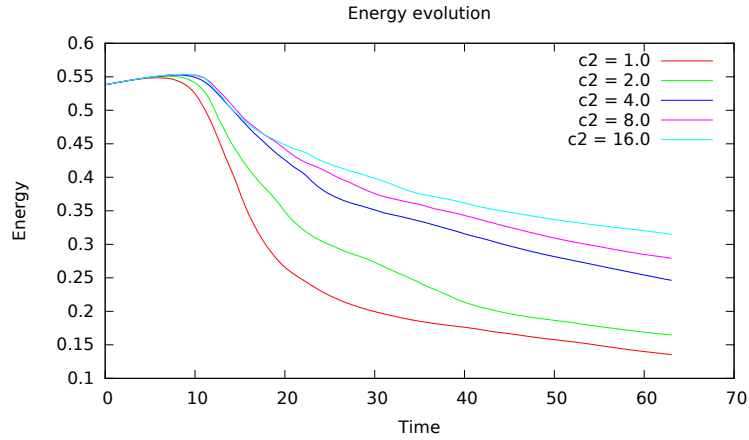

(a) Energy evolution

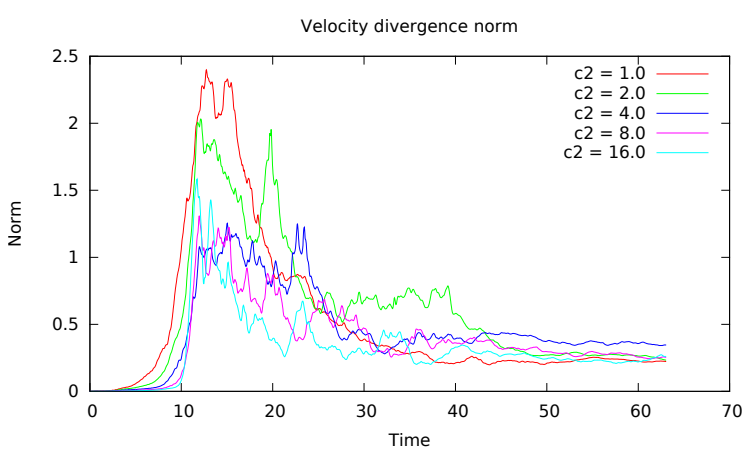

(b) $\left\|\nabla \cdot \mathbf{u}_{h}\right\|$

Figure 14: Energy evolution and velocity divergence norm for different values of $c_{2}$, keeping $c_{1}=12.0$ and $c_{c}=1.0$

It is clearly seen in Fig. 14 that the modification of $c_{2}$ has an effect on the solution. We see in Fig. 14(a) that the energy drops faster when lower values of $c_{2}$ are used. Looking at Fig. 14(b) we see that the velocity divergence $L_{2}$-norm is higher when lower values of $c_{2}$ are taken. The evolution of $\left\|\nabla \cdot \mathbf{u}_{h}\right\|$ gives us information about how the flow is evolving. From an initial and structured condition, the flow starts becoming chaotic around $t=10$, depending on the case, when the turbulent structures increase the rotation of the flow particles, growing the spatial derivatives. At this stage, the energy dissipates until the equilibrium between the internal energy and the external forces is reached. These stages can be seen in Fig. 15, where we depict the vorticity isosurfaces for $|\omega|=5.0$ coloured with the velocity field module at $t=0.15$ (Fig. 15(a)), at $t=12.0$ (Fig. 15(b)) and at $t=70.0$ (Fig. 15(d)) setting $c 2=1.0$ and $c_{c}=32.0$, and using a $32^{3} Q 2 / Q 1$ elements mesh. 